\title{
Distribución del cuadrado de la máxima correlación canónica para tamaños muestrales pequeños
}

\author{
MARÍA R. SANTILLÁN y ALDO J. VIOLLAZ
}

\begin{abstract}
Assume that the normally distributed random vector $\underset{\sim}{X}$ of $d$ components is partitioned into two subvectors $X^{(1)}$ and $X^{(2)}$ of $p$ and $q$ components respectively. Suppose also that the two subvectors are not correlated. In this work we study the distribution of the largest squared canonical correlation $r_{\mathrm{I}}^{2}$ when $p, q$ and the number of observations in the sample $N$, are rather small. We give the cxplicit expressions of the cumulative distribution functions and the computed values of the sample mean and variance of $r_{1}{ }^{2}$. We prove that there exists a stochastic order between the largest squared canonical correlations obtained from two different partitions of the vector $X$. More precisely, $r_{1}{ }^{2}$ increase stochastically when the difference between $p$ and $\tilde{q}$ decreasc. Since $X^{(1)}$ and $X^{(2)}$ are uncorrelated the largest squared canonical correlation in the population $\lambda_{\mathrm{I}}{ }^{2}$ is zero. Therefore the mean of $r_{1}{ }^{2}$ is the bias of $r_{1}{ }^{2}$ when $r_{1}{ }^{2}$ is used to estimate $\lambda_{1}{ }^{2}$. The values of the mean and the variance show that the square of the bias is bigger than the variance in all the cases.
\end{abstract}

\section{INTRODUCCION}

Sea $X$ un vector aleatorio d-dimensional con distribución normal multivariante $N(\mu, \underset{\sim}{\Sigma})$ no singular. Supongamos $\underset{\sim}{X}$ particionado en dos subvectores $X^{(1)} \tilde{y}_{\sim^{(2)}}^{X}$ de dimensiones $p$ y $q(p \leq q, \tilde{p}+q=d)$ respectivamente,

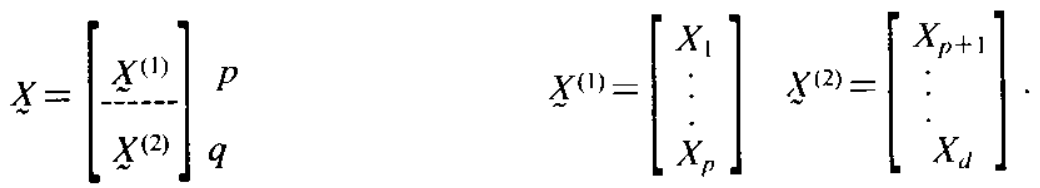

1980 Mathematics Subject Classification (1985 revision): $62 \mathrm{H10}, 62 \mathrm{H2O}$.

Editorial de la Universidad Complutense. Madrid, 1989. 
Esta partición genera una partición de la matriz $\underset{\sim}{\sum}$ en cuatro submatrices:

$$
\underset{\sim}{\Sigma}=\left[\begin{array}{c|c}
\sum_{11} & \sum_{12} \\
\hdashline \sum_{21} & \underset{q}{\sum_{22}}
\end{array}\right]_{q}^{p}
$$

Como estamos interesados en correlaciones, sin pérdida de generalidad, supondremos que $\mu=0$. Como nos ocuparemos del estudio de la distribución muestral de la máxima correlación canónica en el caso en que los dos subvectores $X^{(1)}$ y $X^{(2)}$ son no correlacionados (caso nulo), supondremos que $\Sigma_{12}=\Sigma_{21}=O$. Puesto que las correlaciones canónicas son invariantes bajo transformaciones lineales no singulares, $U=A X^{(1)}$ y $V=B X_{\sim}^{(2)}$ (Kshirsagar 1972), podemos suponer que en [1.2] $\sum_{\sim 1}=I_{p}$ y $\tilde{\Sigma}_{22}=\tilde{I}_{q}$.

Sean $\underset{\sim}{X}, X_{2}, \ldots, \underset{\sim}{X}, N$ observaciones independientes de $\underset{\sim}{X}(N>d)$ y sea $\underset{\sim}{\hat{\Sigma}}$ el estimador máximo verosimil de $\sum_{\tilde{N}}$ particionado en cuatro submatrices en correspondencia a la partición en la población dado por:

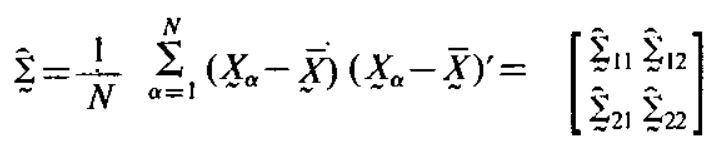

Los estimadores de máxima verosimilitud de los cuadrados de las correlaciones canónicas $\lambda_{1}{ }^{2} \geq \lambda_{2}{ }^{2} \geq \ldots \geq \lambda_{p}^{2}$, son las raíces $r_{1}{ }^{2}>r_{2}{ }^{2}>\ldots>r_{p}{ }^{2}$ de la ecuación determinantal (Hotelling 1939, Kshirsagar 1972, Muirhead 1982, Anderson 1984):

$$
\left|\hat{\Sigma}_{12} \hat{\Sigma}_{22}^{-1} \hat{\Sigma}_{21}-r^{2} \hat{\Sigma}_{11}\right|=0 \text {. }
$$

Las raíces de [1.4] en el caso nulo, bajo el supuesto de normalidad, tienen una đistribución conjunta conocida (Hsu 1939, Girshick 1939). Nosotros estamos interesados en el estudio de la distribución de la máxima de ellas: $r_{1}{ }^{2}$. El análisis de esta distribución es importante, pues cuando el tamaño muestral es relativamente pequeño los estimadores son, como mostraremos, fuertemente sesgados, lo cual puede conducir a conclusiones erróneas, sobre todo cuando se usa la técnica de correlaciones canónicas como una herramienta del análisis exploratorio de datos.

\section{DISTRIBUCION DE LA MAXIMA CORRELACION CANONICA}

La función de densidad conjunta de los cuadrados de las correlaciones canónicas muestrales $r_{1}^{2}>r_{2}^{2}>\ldots>r_{p}^{2}$, en el caso nulo (Anderson 1984, Muirhead 1982, Krishnaiah 1980), viene dada por: 


$$
\begin{gathered}
f\left(r_{1}^{2}, \ldots, r_{p}^{2}\right)=C_{1} \prod_{i=1}^{p}\left[\left(r_{i}^{2}\right)^{(q-p-1) / 2}\left(1-r_{i}^{2}\right)^{(N-d-2) / 2}\right] \prod_{i<j}^{p}\left(r_{i}^{2}-r_{j}^{2}\right), \\
0 \leq r_{p}^{2}<\ldots<r_{1}^{2} \leq 1
\end{gathered}
$$

donde $C_{1}=C_{1}(p, q, N)$ es una constante.

El coeficiente de correlación múltiple $\bar{R}$ es un caso particular de correlación canónica que se obtiene cuando $p=1$. El coeficiente de correlación múltiple muestral al cuadrado $R^{2}$, en el caso nulo $(\bar{R}=0)$ bajo el supuesto de normalidad, tiene una distribución Beta $\left(\frac{d-1}{2}, \frac{N-d}{2}\right.$ ) (Muirhead 1982, Anderson 1984). La función de la densidad de $R^{2}$ puede obtenerse también de [2.1] considerando $p=1, q=d-1$.

En principio de [2.1] se puede obtener la distribución de $r_{1}^{2}$ mediante integración. Sin embargo, en general las funciones de distribución y de densidad de $r_{1}^{2}$ tienen la forma de series infinitas (Pillai 1956, 1967, Sugiyama 1967). En el caso particular en el cual $t=(N-d-2) / 2$ es un entero no negativo, la función de distribución de $r_{1}^{2}$ se puede expresar como un polinomio (Constantine 1963, Muirhead 1982) como sigue:

$$
F(x)=P\left(r_{1}^{2} \leq x\right)=x^{(p q) / 2} \sum_{k=0}^{p t} \frac{(1-x)^{k}}{k !} \sum_{*}^{*}(q / 2)_{\kappa} C_{\kappa}\left(I_{p}\right), 0 \leq x \leq 1,
$$

donde $\kappa$ es la partición del entero $k$ en $p$ partes,

$$
\kappa=\left(k_{1}, k_{2}, \ldots, k_{p}\right)
$$

tales que $k_{1} \geq k_{2} \ldots \geq k_{p}, \sum_{i=1}^{p} k_{i}=k ; 2_{\kappa}^{*}$ denota la suma sobre todas particiones $\kappa$ con la mayor parte $k_{\mathrm{l}} \leq t ;(q / 2)_{\kappa}$ es el coeficiente hipergeométrico generalizado definido por:

$$
(q / 2)_{\kappa}=\prod_{i=1}^{p}((q-i+1) / 2)_{k_{i}}
$$

donde $(\alpha)_{k}$ es el factorial ascendente de $\alpha$ definido para $k$ entero positivo por:

$$
(\alpha)_{k}=\alpha(\alpha+1) \ldots(\alpha+k-1)
$$

y para $k=0$ por $(\alpha)_{0}=1$ donde $\alpha$ es un real arbitrario. Si $\alpha$ es un entero negativo y $k>|\alpha|$ entonces $(\alpha)_{k}=0 . C_{k}\left(I_{p}\right)$ es el llamado polinomio zonal asociado a $I_{p}$ (James 1960, 1961a, 1961b, 1964, 1968, Constantine 1963, 1966, Muirhead 1982) que se calcula para $2 \leq m \leq p$ como: 


$$
C_{\kappa}\left(I_{p}\right)=2^{2 k} k(p / 2)_{\kappa} \frac{\prod_{i<j}^{m}\left(2 k_{j}-2 k_{j}-i+j\right)}{\prod_{i=1}^{m}\left(2 k_{i}+m-i\right) !}
$$

donde $m$ es el número de partes no nulas de la partición $\kappa$, y para $m=1$ como:

$$
C_{k}\left(I_{p}\right)=\left(\operatorname{tr}\left(I_{p}\right)\right)^{k}-\underset{\kappa \neq\left(k_{\ldots} \ldots, 0\right)}{\sum_{k} C_{k}\left(I_{p}\right)}
$$

Definiendo $b=(p q) / 2$ y $a_{k}$ para $k=0,1, \ldots, p t$ por

$$
a_{k}=\Sigma_{\kappa}^{*}(q / 2)_{\kappa} C_{\kappa}\left(I_{p}\right) / k !
$$

Tabla 1

Coeficientes de la función de distribución del cuadrado de la máxima correlación

\begin{tabular}{|c|c|c|c|c|c|c|c|c|c|c|}
\hline$N$ & $P$ & $b$ & $u_{0}$ & $\begin{array}{l}a_{1} \\
a_{8}\end{array}$ & $\begin{array}{l}a_{2} \\
a_{9}\end{array}$ & $\begin{array}{c}a_{3} \\
a_{10}\end{array}$ & $\begin{array}{c}a_{4} \\
a_{11}\end{array}$ & $\begin{array}{c}a_{5} \\
a_{12}\end{array}$ & $\begin{array}{c}a_{6} \\
a_{13}\end{array}$ & $\begin{array}{c}a_{7} \\
a_{14}\end{array}$ \\
\hline 6 & \multirow{8}{*}{ l } & $3 / 2$ & 1.00 & & & & & & & \\
\hline 8 & & $3 / 2$ & 1.00 & 1.50 & & & & & & \\
\hline 10 & & $3 / 2$ & 1.00 & 1.50 & 1.88 & & & & & \\
\hline 12 & & $3 / 2$ & 1.00 & 1.50 & 1.88 & 2.19 & & & & \\
\hline 14 & & $3 / 2$ & 1.00 & 1.50 & 1.88 & 2.19 & 2.46 & & & \\
\hline 16 & & $3 / 2$ & 1.00 & 1.50 & 1.88 & 2.19 & 2.46 & 2.71 & & \\
\hline 18 & & $3 / 2$ & 1.00 & 1.50 & 1.88 & 2.19 & 2.46 & 2.71 & 2.93 & \\
\hline 20 & & $3 / 2$ & 1.00 & 1.50 & 1.88 & 2.19 & 2.46 & 2.71 & 2.93 & 3.14 \\
\hline 6 & \multirow{10}{*}{2} & 2 & 1.00 & & & & & & & \\
\hline 8 & & 2 & 1.00 & 2.00 & 0.33 & & & & & \\
\hline 10 & & 2 & 1.00 & 2.00 & 3.00 & 0.80 & 0.20 & & & \\
\hline 12 & & 2 & 1.00 & 2.00 & 3.00 & 4.00 & 1.34 & 0.51 & 0.14 & \\
\hline 14 & & 2 & 1.00 & $\begin{array}{l}2.00 \\
0.11\end{array}$ & 3.00 & 4.00 & 5.00 & 1.94 & 0.90 & 0.38 \\
\hline 16 & & 2 & 1.00 & $\begin{array}{l}2.00 \\
0.69\end{array}$ & $\begin{array}{l}3.00 \\
0.30\end{array}$ & $\begin{array}{l}4.00 \\
0.09\end{array}$ & 5.00 & 6.00 & 2.57 & 1.35 \\
\hline 18 & & 2 & 1.00 & 2.00 & 3.00 & 4.00 & 5.00 & 6.00 & 7.00 & 3.23 \\
\hline & & & & 1.84 & 1.05 & 0.56 & 0.25 & 0.08 & & \\
\hline 20 & & 2 & 1.00 & 2.00 & 3.00 & 4.00 & 5,00 & 6.00 & 7.00 & 8.00 \\
\hline & & & & 3.91 & 2.36 & 1.45 & 0.86 & 0.47 & 0.22 & 0.07 \\
\hline
\end{tabular}
canónica muestral en el caso nulo $F(x ; p, q, N)=x^{b}\left(a_{0}+a_{l}(l-x)+\ldots+a_{k}(l-x)^{k}\right)$, para $d=p+q=4$ 
se encuentra fácilmente que $a_{0}=1$ y $a_{1}=b$. Por lo tanto [2.2] puede escribirse en la forma:

$$
F(x)=F(x ; p, q, N)=x^{b}\left[1+b(1-x)+\ldots+a_{p t}(1-x)^{m t}\right], 0 \leq x \leq 1
$$

Las Tablas 1, 2 y 3 dan los valores de los coeficientes de $F(x)$ para valores de $N \leq 21, d=4,5,6$ y $p=1,2,3$, que de acuerdo a las definiciones [2.4], [2.5], [2.6] y [2.7], resultan números racionales, pero que por razones de presentación se expresan en forma decimal. En el Apéndice se dan tablas más extensas que llegan hasta $N=40$ y $d=7$.

Tabla 2

Coeficientes de la función de distribución del cuadrado de la máxima correlación canónica muestral en el caso nulo, para $d=p+q=5$

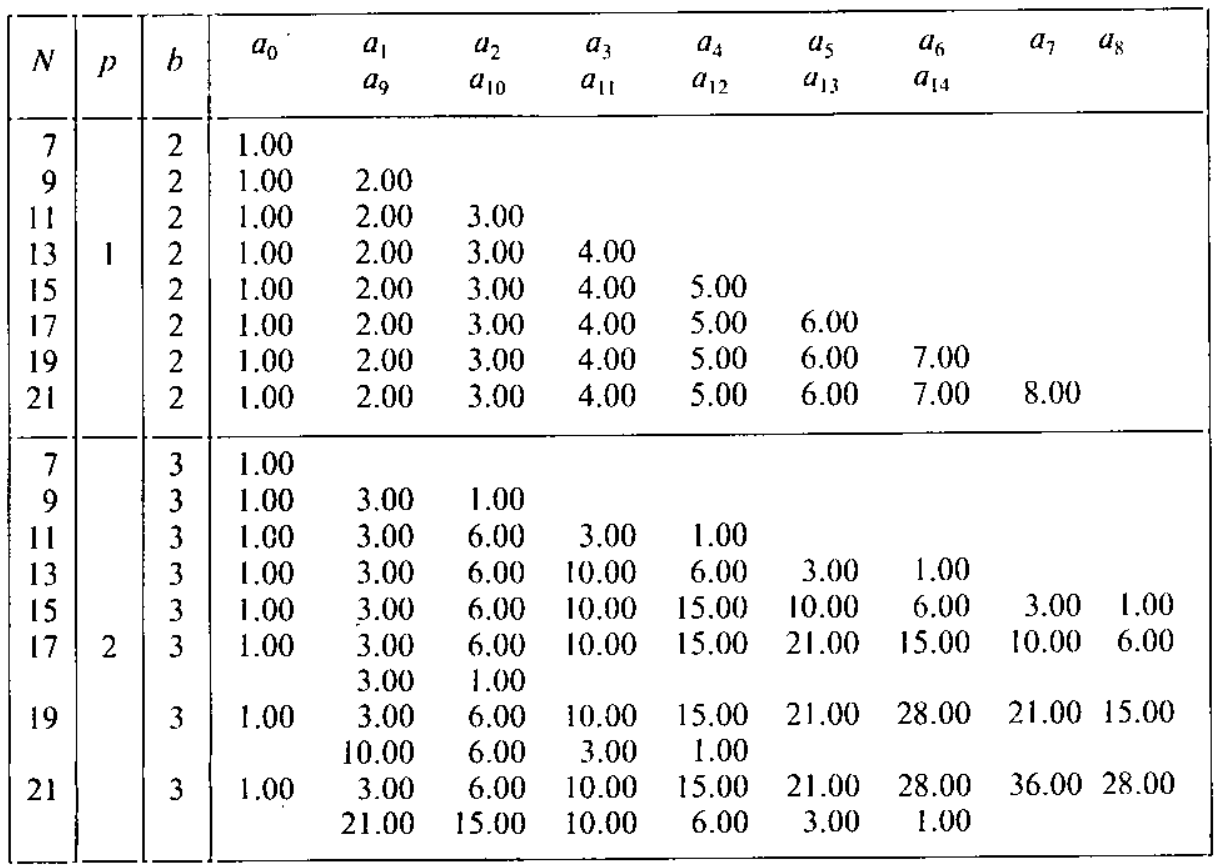


Tabla 3

Coeficientes de la función de distribución del cuadrado de la máxima correlación canónica muestral en el caso mulo, para $d=p+q=6$

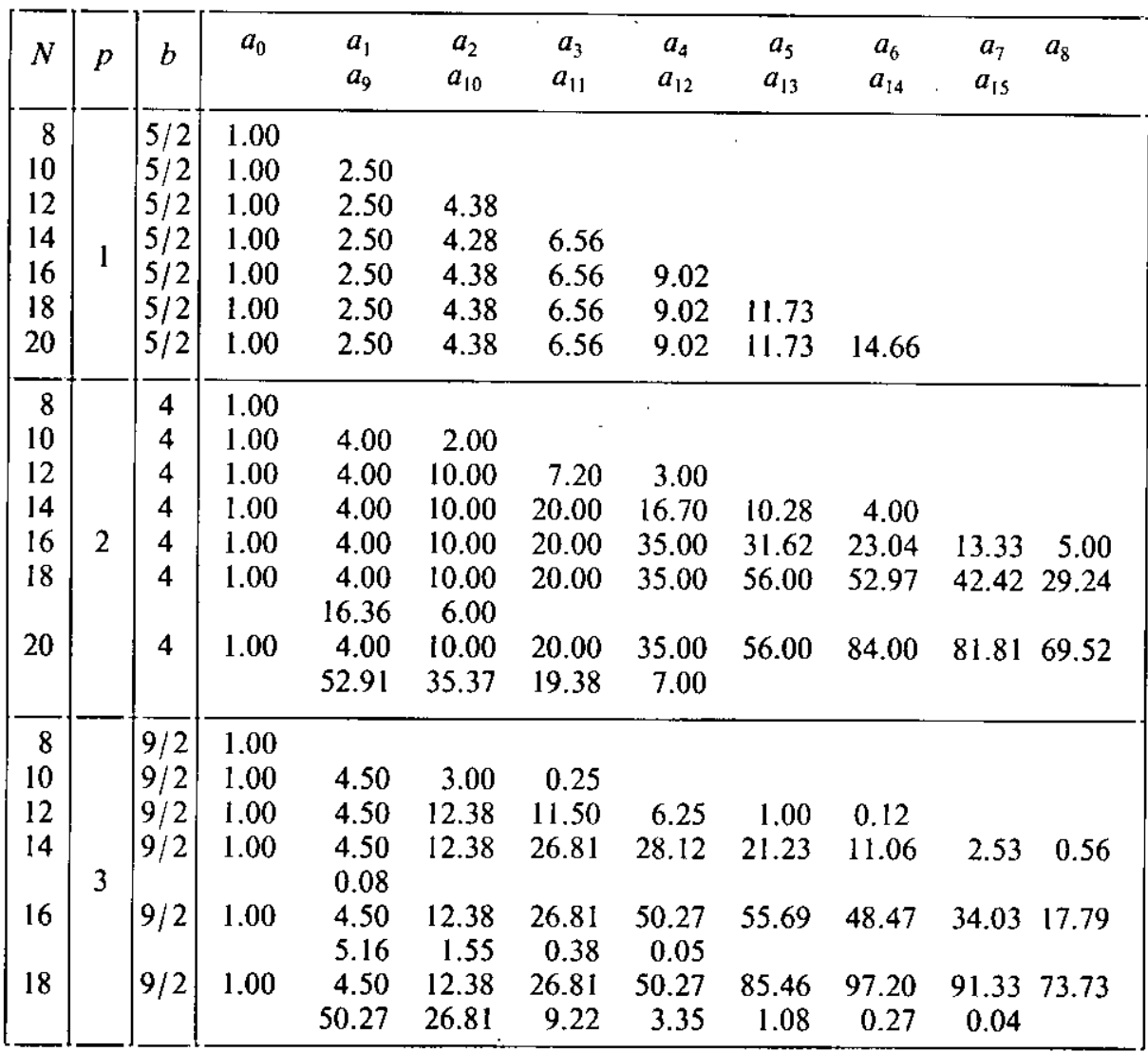

\section{COMPUTO DE LOS COEFICIENTES DE LA FUNCION DE DISTRIBUCION}

Utilizando las expresiones [2.3]-[2.8] se ha realizado el cómputo de los coeficientes de la función de distribución $\bar{F}(x)=\bar{F}(x ; p, q, N)$, expresada en la forma [2.9], para $d=4,5,6,7, p=1,2,3$ y valores de $N \leq 40$, sujeto a la condición que $t=(N-d-2) / 2$ sea un entero no negativo.

El cálculo de los coeficientes $a_{k}$ resulta engorroso aún para valores relativamente pequeños de $N$ y $d$. La complejidad en el cálculo se ve claramente si se analizan las definiciones [2.4]-[2.7]. En algunos casos sencillos 
como $p=1$ es fácil determinar en forma explícita $a_{k}$ para $k=0,1, \ldots, p t$ dado por [2.8]. En ese caso particular, $\kappa=k$ y por lo tanto, $(q / 2)_{\kappa}=((p-1) / 2)_{k}$ en [2.4] y $C_{k}\left(I_{p}\right)=1$ en [2.7], resultando $a_{k}=((p-1) / 2)_{k}$. Para $p \geq 2$ [2.4] tiene como máximo $p$ factores de la forma $(\alpha)_{k}$, que a partir de ciertos valores de $N$, para un determinado valor de $d$, hacen que $(q / 2)_{\kappa}$ y también $C_{\kappa}\left(I_{p}\right)$ sean cocientes de números enteros muy grandes. En consecuencia se hace necesario calcular $a_{k}$ mediante programas de cómputos que tengan en cuenta los problemas de cálculo numérico originados al operar con números de gran magnitud.

El programa principal de cómputo desarrollado se basa en dos subrutinas que calculan los coeficientes hipergeométricos generalizados [2.4] y los polinomios zonales [2.6]. Para cada valor de $k=0,1, \ldots, p t$ se consideran aquellas particiones $\kappa$ definidas en [2.3] que cumplen con $k_{1} \leq t$. Con ellas se calcula un coeficiente que depende de $\kappa, \operatorname{COEF}(\kappa)=(q / 2)_{\kappa} C_{k}\left(I_{p}\right) / k !$ Luego para el valor de $k$ considerado se realiza la suma de los coeficientes $\operatorname{COEF}(\kappa)$ para obtener $a_{k}$ como se define en [2.8].

Con el mismo programa se evalúan los valores esperados y las varianzas de la máxima correlación canónica $r_{t}^{2}$ que se presentan en la Sección 5.

\section{RELACIONES DE ORDEN ESTOCASTICO}

La máxima correlación canónica muestral puede interpretarse como el coseno del ángulo que forman los subespacios vectoriales generados por los dos grupos de variables. Esta interpretación geométrica nos lleva a conjeturar que la máxima correlación canónica debe crecer estocásticamente a medida que las dimensiones $p$ y $q$ se aproximan entre sí manteniéndose constante $d=p+q$

Sean $p, p^{\prime}, q, q^{\prime}$, enteros positivos tales que $p+q=p^{\prime}+q^{\prime}, p<q, p^{\prime} \leq q^{\prime}$, $p<p^{r}$ y denotemos con $r_{1}^{2}(p, q, N)$ y con $F(x ; p, q, N)$ la máxima correlación canónica al cuadrado y su función de distribución, respectivamente. Nuestra conjetura es que

$$
r_{1}^{2}(p, q, N) \leq r_{1}^{2}\left(p^{\prime}, q^{\prime}, N\right)
$$

o equivalentemente

$$
F(x ; p, q, N) \geq F\left(x ; p^{\prime}, q^{\prime}, N\right)
$$

Utilizando la notación de la Sección 2 la ecuación [4.2] puede escribirse como

$$
\frac{F(x ; p, q, N)}{F\left(x ; p^{\prime}, q^{\prime}, N\right)}=\frac{x^{b}\left(a_{0}+a_{1}(1-x)+\ldots+a_{f}(1-x)^{r}\right)}{x^{b^{\prime}}\left(a_{0}^{\prime}+a_{1}^{\prime}(1-x)+\ldots+a_{s}^{\prime}(1-x)^{s}\right)} \geq 1
$$


donde $a_{0}=a_{0}^{\prime}=1, b=p q / 2, b^{\prime}=p^{\prime} q^{\prime} / 2$. Nótese que si $d=p+q=p^{\prime}+q^{\prime}$ es un número impar $b^{\prime}-b$ es un entero positivo y si $d$ es par $b^{\prime}-b$ es un múltiplo de 1/2. La siguiente proposición es la clave para la demostración de la validez de la desigualdad [4.3].

Proposición 4.1. Sean $\sum_{k=0}^{\infty} a_{k}(I-x)^{k}, \sum_{k=0}^{\infty} b_{k}(I-x)^{\mathrm{k}}$ series convergentes para $0 \leq x \leq 1$, con $a_{0}=b_{0}=1, a_{k} \geq 0, b_{k} \geq 0, k=1,2, \ldots$ Entonces condición suficiente para que

$$
\frac{\sum_{k=0}^{\infty} a_{k}(1-x)^{k}}{x \sum_{k=0}^{\infty} b_{x}(1-x)^{k}} \geq 1,0 \leq x \leq 1
$$

es que

$$
b_{k} \leq b_{k-1}+a_{k}, k=1,2, \ldots,
$$

o equivalentemente

$$
b_{k} \leq a_{0}+\ldots+a_{k}, k=I, 2, \ldots
$$

Demostración. La inecuación [4.4] es equivalente a

$$
\sum_{k=0}^{\infty} a_{k}(1-x)^{k} \geq((x-1)+1) \sum_{k=0}^{\infty} b_{k}(1-x)^{k}
$$

la que a su vez es equivalente a

$$
a_{0}+\sum_{k=1}^{\infty}\left(a_{k}+b_{k-1}\right)(1-x)^{k} \geq \sum_{k=0}^{\infty} b_{k}(1-x)^{k}
$$

Para que [4.7] sea verdadera es suficiente que

$$
b_{0} \leq a_{0}, b_{k} \leq b_{k-1}+a_{k}, k=1,2, \ldots
$$

La desigualdad [4.6] se sigue de inmediato de [4.5].

Mediante el empleo iterado de la Proposición 4.1 se estableció la validez de [4.3] en el caso en que $b^{\prime}-b$ es un entero positivo. Cuando $b^{\prime}-b$ es un múltiplo de $1 / 2$ se consideró el cuadrado de [4.3] y luego se aplicó la Proposición 4.1. 
Con el método descripto se ha probado la validez de [4.2] para todas las funciones de distribución tabuladas en este trabajo. Establecer la validez del orden estocástico para valores arbitrarios de $p, q$ y $N$ es un problema difícil, pues no se conoce una forma general tratable de los coeficientes de $F(x)$.

Vale la pena hacer notar que para los casos estudiados las desigualdades [4.6] resultaron frecuentemente igualdades para los coeficientes de grados menores. (Ver tablas del Apéndice.).

Los valores de las esperanzas matemáticas de $r_{1}^{2}$ calculados en la Sección 5 están de acuerdo con el orden estocástico y pueden tomarse como una medida global del mismo.

\section{SESGO Y VARIANZA}

Las expresiones para la media y la varianza del coeficiente de correlación múltiple muestral $R$ y de su cuadrado $R^{2}$, en el caso nulo, se pueden calcular a partir de la función de densidad de $R^{2}$, siendo iguales a:

$$
\begin{array}{ll}
E(R)=\frac{\Gamma\left(\frac{1}{2}(N-1)\right) \Gamma\left(\frac{1}{2} d\right)}{\Gamma\left(\frac{1}{2}(d-1)\right) \Gamma\left(\frac{1}{2} N\right)} & \operatorname{Var}(R)=\frac{d-1}{N-1}-[E(R)]^{2} \\
E\left(R^{2}\right)=\frac{d-1}{N-1} & \operatorname{Var}\left(R^{2}\right)=\frac{2(d-1)(N-d)}{(N-1)\left(N^{2}-1\right)}
\end{array}
$$

No son conocidas expresiones exactas para la media y la varianza de la máxima correlación canónica muestral ni de su cuadrado, cuando $p>1$. Existen expresiones asintóticas para la media y la varianza de la máxima correlación canónica muestral en el caso no nulo (Lawley 1959).

En este trabajo hemos calculado la media y la varianza de $r_{1}^{2}$ en base a las funciones de distribución encontradas en la Sección 2, mediante las expresiones:

$$
\begin{array}{r}
E\left(r_{1}^{2}\right)=1-\int_{0}^{1} F(x) d x=1-\sum_{k=0}^{m t} a_{k} \frac{(1)_{k}}{\left(\frac{p q}{2}+1\right)_{k}+1} \\
E\left(r_{1}^{4}\right)=1-2 \int_{0}^{1} x F(x) d x=1-2 \sum_{k=1}^{p t} a_{k} \frac{(1)_{k}}{\left(\frac{p q}{2}+2\right)_{k+1}}
\end{array}
$$




$$
\operatorname{Var}\left(r_{1}^{2}\right)=E\left(r_{1}^{4}\right)-\left(E\left(r_{1}^{2}\right)\right)^{2}
$$

teniendo en cuenta que $\int_{0}^{1} x^{\alpha-1} \quad(1-x)^{\beta-1} d x=\Gamma(\alpha) \Gamma(\beta) / \Gamma(\alpha+\beta)$ y $\Gamma(\alpha+k) / \Gamma(\alpha)=(\alpha)_{k}$ para $\alpha>0$. La Tabla 4 muestra los valores de la media y la desviación estándar de $r_{\mathrm{l}}^{2}$ para distintos valores de $d, p$ y $N$.

Tabla 4

Media y desviación estándar del cuadrado de la máxima correlación canónica muestral para $d=p+q=4,5,6$

\begin{tabular}{|c|c|c|c|c|c|c|c|c|c|c|}
\hline$d$ & $p$ & $N$ & 6 & 8 & 10 & 12 & 14 & 16 & 18 & 20 \\
\hline \multirow{2}{*}{4} & 1 & $\frac{\text { Media }}{\sqrt{\text { Var }}}$ & $\begin{array}{l}0.600 \\
0.263\end{array}$ & $\begin{array}{l}0.429 \\
0.232\end{array}$ & $\begin{array}{l}0.333 \\
0.200\end{array}$ & $\begin{array}{l}0.273 \\
0.173\end{array}$ & $\begin{array}{l}0.231 \\
0.155\end{array}$ & $\begin{array}{l}0.200 \\
0.138\end{array}$ & $\begin{array}{l}0.176 \\
0.122\end{array}$ & $\begin{array}{l}0.158 \\
0.114\end{array}$ \\
\hline & 2 & $\frac{\text { Media }}{\sqrt{\text { Var }}}$ & $\begin{array}{l}0.667 \\
0.235\end{array}$ & $\begin{array}{l}0.489 \\
0.224\end{array}$ & $\begin{array}{l}0.385 \\
0.197\end{array}$ & $\begin{array}{l}0.317 \\
0.173\end{array}$ & $\begin{array}{l}0.269 \\
0.155\end{array}$ & $\begin{array}{l}0.234 \\
0.138\end{array}$ & $\begin{array}{l}0.207 \\
0.127\end{array}$ & $\begin{array}{l}0.190 \\
0.114\end{array}$ \\
\hline
\end{tabular}

\begin{tabular}{|c|c|c|c|c|c|c|c|c|c|c|}
\hline$d$ & $p$ & $N$ & 7 & 9 & 11 & 13 & 15 & 17 & 19 & 21 \\
\hline \multirow{4}{*}{5} & 1 & Media & 0.667 & 0.500 & 0.400 & 0.333 & 0.286 & 0.250 & 0.222 & 0.200 \\
\hline & & $\sqrt{ }$ Var & 0.237 & 0.224 & 0.200 & 0.179 & 0.158 & 0.145 & 0.130 & 0.122 \\
\hline & 2 & Media & 0.750 & 0.583 & 0.475 & 0.400 & 0.345 & 0.304 & 0.271 & 0.244 \\
\hline & 2 & $\sqrt{\text { Var }}$ & 0.192 & 0.202 & 0.187 & 0.173 & 0.158 & 0.145 & 0.130 & 0.122 \\
\hline
\end{tabular}

\begin{tabular}{|c|c|c|c|c|c|c|c|c|c|c|}
\hline$d$ & $p$ & $N$ & 8 & 10 & 12 & 14 & 16 & 18 & 20 & - \\
\hline \multirow{5}{*}{6} & 1 & $\frac{\text { Media }}{\sqrt{V_{a r}}}$ & $\begin{array}{l}0.714 \\
0.212\end{array}$ & $\begin{array}{l}0.556 \\
0212\end{array}$ & $\begin{array}{l}0.455 \\
0.195\end{array}$ & $\begin{array}{l}0.385 \\
0.179\end{array}$ & $\begin{array}{l}0.333 \\
0.161\end{array}$ & $\begin{array}{l}0.294 \\
0.148\end{array}$ & 0.263 & - \\
\hline & \multirow{2}{*}{2} & Media & 0.800 & 0.648 & 0.541 & 0.464 & 0405 & 0360 & 0323 & - \\
\hline & & $\sqrt{\mathrm{Var}}$ & 0.164 & 0.182 & 0.176 & 0.167 & 0.155 & 0.141 & 0.134 & - \\
\hline & \multirow{2}{*}{3} & Media & 0.818 & 0.669 & 0.562 & 0.484 & 0.424 & 0.377 & - & - \\
\hline & & $\sqrt{\text { Var }}$ & 0.152 & 0.173 & 0.170 & 0.161 & 0.152 & 0.141 & - & - \\
\hline
\end{tabular}

Puesto que el coeficiente de correlación canónico máximo de la población $\lambda_{1}$ es cero ( $X^{(1)}$ y $X^{(2)}$ son no correlacionadas), la media de $r_{1}^{2}$ es el sesgo de $r_{1}^{2}$ como estimador de $\lambda_{1}^{2}=0$. La Tabla 4 muestra que en todos los casos el sesgo es sensiblemente más grande que la desviación estándar, es decir, en el error cuadrático medio de $r_{1}^{2}$ como estimador de $\lambda_{1}^{2}$, el sesgo aporta la contribución mayor.

Para muestras más grandes que las estudiadas, o aún asintóticamente, no se conoce cuál es la magnitud relativa entre el cuadrado del sesgo y la varianza. 


\section{CONCLUSIONES}

Este trabajo constituye una primera aproximación al problema de determinar la distribución de probabilidad, la media y la varianza del cuadrado de la máxima correlación canónica y establecer la existencia de una relación de orden estocástico. La prueba de la validez de la relación de orden estocástico, en general, sería de utilidad pues entonces todas las funciones de distribución estarian comprendidas entre la función de distribución de la correlación canónica correspondientes a grupos donde $p=[d / 2]$, la parte entera de $d / 2$, y la función de distribución del coeficiente de correlación múltiple, es decir,

$$
F(x ;[d / 2], d-[d / 2], N) \leq F(x ; p, q, N) \leq F(x ; 1, d-1, N)
$$

En este trabajo hemos calculado la media y la varianza del cuadrado de la máxima correlación canónica muestral y hemos establecido la validez de la relación de orden estocástico para tamaños muestrales menores o iguales a 40 y dimensión $d=p+q$ menor o igual a 7 . Estos cálculos muestran que el sesgo del cuadrado de la máxima correlación canónica muestral es sensiblemente mayor que la correspondiente desviación estándar.

Debido al pequeño tamaño muestral, los resultados obtenidos son limitados, pero fundamentalmente deberían constituir un toque de atención para el usuario de las correlaciones canónicas, sobre todo cuando se las usa como herramienta del análisis exploratorio de datos.

\section{APENDICE}

En este apéndice se presentan tablas conteniendo los coeficientes de las funciones de distribución, media y desviación estándar del cuadrado de la máxima correlación canónica muestral en el caso nulo para $d=4,5,6,7$ y tamaños muestrales menores o iguales a 40 . 
Tabla A, 1

Coeficientes de la función de distribución del cuadrado de la máxima correlación canónica muestral en el caso nulo, $F(x ; p, q, n)=x^{h}\left(a_{0}+a_{1}(1-x)+\ldots+a_{k}(1-x)^{k}\right)$ para $d=p+q=4$

\begin{tabular}{|c|c|c|c|c|c|c|c|c|c|c|c|c|c|c|c|c|c|c|c|c|}
\hline$n$ & $p$ & $b$ & $a_{0}$ & $a_{1}$ & $a_{2}$ & $a_{3}$ & $a_{4}$ & $a_{5}$ & $a_{6}$ & $a_{7}$ & $a_{8}$ & $a_{9}$ & $a_{10}$ & $a_{1 \mid}$ & $a_{12}$ & $a_{13}$ & $a_{14}$ & $a_{15}$ & $a_{16}$ & $a_{17}$ \\
\hline 6 & & $3 / 2$ & 1.00 & & & & & & & & & & & & & & & & & \\
\hline 8 & & $3 / 2$ & 1.00 & 1.50 & & & & & & & & & & & & & & & & \\
\hline 10 & & $3 / 2$ & 1.00 & 1.50 & 1.88 & & & & & & & & & & & & & & & \\
\hline 12 & & $3 / 2$ & 1.00 & 1.50 & 1.88 & 2.19 & & & & & & & & & & & & & & \\
\hline 14 & & $3 / 2$ & 1.00 & 1.50 & 1.88 & 2.19 & 2.46 & & & & & & & & & & & & & \\
\hline 16 & & $3 / 2$ & 1.00 & 1.50 & 1.88 & 2.19 & 2.46 & 2.71 & & & & & & & & & & & & \\
\hline 18 & & $3 / 2$ & 1.00 & 1.50 & 1.88 & 2.19 & 2.46 & 2.71 & 2.93 & & & & & & & & & & & \\
\hline 20 & 1 & $3 / 2$ & 1.00 & 1.50 & 1.88 & 2.19 & 2.46 & 2.71 & 2.93 & 3.14 & & & & & & & & & & \\
\hline 22 & & $3 / 2$ & 1.00 & 1.50 & 1.88 & 2.19 & 2.46 & 2.71 & 2.93 & 3.14 & 3.34 & & & & & $=$ & & & & \\
\hline 24 & & $3 / 2$ & 1.00 & 1.50 & 1.88 & 2.19 & 2.46 & 2.71 & 2.93 & 3.14 & 3.34 & 3.52 & & & & & & & & \\
\hline 26 & & $3 / 2$ & 1.00 & 1.50 & 1.88 & 2.19 & 2.46 & 2.71 & 2.93 & 3.14 & 3.34 & 3.52 & 3.70 & & & & & & & \\
\hline 28 & & $3 / 2$ & 1.00 & 1.50 & 1.88 & 2.19 & 2.46 & 2.71 & 2.93 & 3.14 & 3.34 & 3.52 & 3.70 & 3.87 & & & & & & \\
\hline 30 & & $3 / 2$ & 1.00 & 1.50 & 1.88 & 2.19 & 2.46 & 2.71 & 2.93 & 3.14 & 3.34 & 3.52 & 3.70 & 3.87 & 4.03 & & & & & \\
\hline 32 & & $3 / 2$ & 1.00 & 1.50 & 1.88 & 2.19 & 2.46 & 2.71 & 2.93 & 3.14 & 3.34 & 3.52 & 3.70 & 3.87 & 4.03 & 4.18 & & & & \\
\hline 34 & & $3 / 2$ & 1.00 & 1.50 & 1.88 & 2.19 & 2.46 & 2.71 & 2.93 & 3.14 & 3.34 & 3.52 & 3.70 & 3.87 & 4.03 & 4.18 & 4.33 & & & \\
\hline 36 & & $3 / 2$ & 1.00 & 1.50 & 1.88 & 2.19 & 2.46 & 2.71 & 2.93 & 3.14 & 3.34 & 3.52 & 3.70 & 3.87 & 4.03 & 4.18 & 4.33 & 4.48 & & \\
\hline 38 & & $3 / 2$ & 1.00 & 1.50 & 1.88 & 2.19 & 2.46 & 2.71 & 2.93 & 3.14 & 3.34 & 3.52 & 3.70 & 3.87 & 4.03 & 4.18 & 4.33 & 4.48 & 4.62 & \\
\hline 40 & & $3 / 2$ & 1.00 & 1.50 & 1.88 & 2.19 & 2.46 & 2.71 & 2.93 & 3.14 & 3.34 & 3.52 & 3.70 & 3.87 & 4.03 & 4.18 & 4.33 & 4.48 & 4.62 & 4.75 \\
\hline
\end{tabular}


Tabla A. 1

(Continuación)

\begin{tabular}{|c|c|c|c|c|c|c|c|c|c|c|c|c|c|c|c|c|c|c|c|c|}
\hline$n$ & $p$ & $b$ & $a_{0}$ & $\begin{array}{c}a_{1} \\
a_{18}\end{array}$ & $\begin{array}{c}a_{2} \\
a_{19}\end{array}$ & $\begin{array}{c}a_{3} \\
a_{20}\end{array}$ & $\begin{array}{r}a_{4} \\
a_{21}\end{array}$ & $\begin{array}{c}a_{5} \\
a_{22}\end{array}$ & $\begin{array}{c}a_{6} \\
a_{23}\end{array}$ & $\begin{array}{c}a_{7} \\
a_{24}\end{array}$ & $\begin{array}{c}a_{8} \\
a_{25}\end{array}$ & $\begin{array}{c}a_{9} \\
a_{26}\end{array}$ & $\begin{array}{l}a_{10} \\
a_{27}\end{array}$ & $\begin{array}{l}a_{11} \\
a_{28}\end{array}$ & $\begin{array}{l}a_{12} \\
a_{29}\end{array}$ & $\begin{array}{l}a_{13} \\
a_{30}\end{array}$ & $\begin{array}{l}a_{14} \\
a_{31}\end{array}$ & $\begin{array}{l}a_{15} \\
a_{32}\end{array}$ & $\begin{array}{l}a_{16} \\
a_{33}\end{array}$ & $\begin{array}{l}a_{17} \\
a_{34}\end{array}$ \\
\hline 6 & & 2 & 1.00 & & & & & & & & & & & & & & & & & \\
\hline 8 & & 2 & 1.00 & 2,00 & 0.33 & & & & & & & & & & & & & & & \\
\hline 10 & & 2 & 1.00 & 2.00 & 3.00 & 0.80 & 0.20 & & & & & & & & & & & & & \\
\hline 12 & & 2 & 1.00 & 2.00 & 3.00 & 4.00 & 1.34 & 0.51 & 0.14 & & & & & & & & & & & \\
\hline 14 & & 2 & 1.00 & 2.00 & 3.00 & 4.00 & 5.00 & 1.94 & 0.90 & 0.38 & 0.11 & & & & & & & & & \\
\hline 16 & & 2 & 1.00 & 2.00 & 3.00 & 4.00 & 5.00 & 6.00 & 2.57 & 1.35 & 0.69 & 0.30 & 0.09 & & & & & & & \\
\hline 18 & & 2 & 1.00 & 2.00 & 3.00 & 4.00 & 5.00 & 6.00 & 7.00 & 3.23 & 1.84 & 1.05 & 0.56 & 0.25 & 0.08 & & & & & \\
\hline 20 & & 2 & 1.00 & 2.00 & 3.00 & 4.00 & 5.00 & 6.00 & 7.00 & 8.00 & 3.91 & 2.36 & 1.45 & 0.86 & 0.47 & 0.21 & 0.07 & & & \\
\hline 22 & & 2 & 1.00 & 2.00 & 3,00 & 4.00 & 5.00 & 6.00 & 7.00 & 8.00 & 9.00 & 4.61 & 2.91 & 1.89 & 1.21 & 0.73 & 0.40 & 0.19 & 0.06 & \\
\hline 24 & & 2 & 1.00 & $\begin{array}{l}2.00 \\
0.05\end{array}$ & 3.00 & 4.00 & 5.00 & 6.00 & 7.00 & 8.00 & 9.00 & 10.00 & 5.32 & 3.49 & 2.36 & 1.58 & 1.03 & 0.64 & 0.36 & 0.17 \\
\hline 26 & 2 & 2 & 1.00 & $\begin{array}{l}2.00 \\
0.32\end{array}$ & $\begin{array}{l}3.00 \\
0.15\end{array}$ & $\begin{array}{l}4.00 \\
0.05\end{array}$ & 5.00 & 6.00 & 7.00 & 8.00 & 9.00 & 10.00 & 11.00 & 6.05 & 4.08 & 2.85 & 1.99 & 1.37 & 0.90 & 0.56 \\
\hline 28 & & 2 & 1.00 & $\begin{array}{l}2.00 \\
0.80\end{array}$ & $\begin{array}{l}3.00 \\
0.51\end{array}$ & $\begin{array}{l}4.00 \\
0.28\end{array}$ & $\begin{array}{l}5.00 \\
0.14\end{array}$ & $\begin{array}{l}6.00 \\
0.04\end{array}$ & 7.00 & 8.00 & 9.00 & 10.00 & 11.00 & 12.00 & 6.80 & 4.69 & 3.37 & 2.43 & 1.73 & 1.20 \\
\hline 30 & & 2 & 1.00 & $\begin{array}{l}2.00 \\
1.53\end{array}$ & $\begin{array}{l}3,00 \\
1.08\end{array}$ & $\begin{array}{l}4.00 \\
0.73\end{array}$ & $\begin{array}{l}5.00 \\
0.46\end{array}$ & $\begin{array}{l}6.00 \\
0.26\end{array}$ & $\begin{array}{l}7.00 \\
0.12\end{array}$ & $\begin{array}{l}8.00 \\
0.04\end{array}$ & 9.00 & 10.00 & 11.00 & 12.00 & 13.00 & 7.55 & 5.32 & 3.90 & 2.88 & 2.12 \\
\hline 32 & & 2 & 1.00 & $\begin{array}{l}2.00 \\
2.53\end{array}$ & $\begin{array}{l}3.00 \\
1.89\end{array}$ & $\begin{array}{l}4.00 \\
1.38\end{array}$ & $\begin{array}{l}5.00 \\
0.98\end{array}$ & $\begin{array}{l}6.00 \\
0.66\end{array}$ & $\begin{array}{l}7.00 \\
0.42\end{array}$ & $\begin{array}{l}8.00 \\
0.24\end{array}$ & $\begin{array}{l}9.00 \\
0.12\end{array}$ & $\begin{array}{r}10.00 \\
0.04\end{array}$ & 11.00 & 12.00 & 13.00 & 14.00 & 8.31 & 5.96 & 4.45 & 3.36 \\
\hline 34 & & 2 & 1.00 & $\begin{array}{l}2.00 \\
3.86\end{array}$ & $\begin{array}{l}3.00 \\
2.97\end{array}$ & $\begin{array}{l}4.00 \\
2.26\end{array}$ & $\begin{array}{l}5.00 \\
1.70\end{array}$ & $\begin{array}{l}6.00 \\
1.25\end{array}$ & $\begin{array}{l}7.00 \\
0.89\end{array}$ & $\begin{array}{l}8.00 \\
0.61\end{array}$ & $\begin{array}{l}9.00 \\
0.39\end{array}$ & $\begin{array}{r}10.00 \\
0.22\end{array}$ & $\begin{array}{r}11.00 \\
0.11\end{array}$ & $\begin{array}{r}12.00 \\
0.03\end{array}$ & 13,00 & 14.00 & 15.00 & 9.08 & 6.61 & 5.02 \\
\hline 36 & & 2 & 1.00 & $\begin{array}{l}2.00 \\
5.60\end{array}$ & $\begin{array}{l}3.00 \\
4.37\end{array}$ & $\begin{array}{l}4.00 \\
3.42\end{array}$ & $\begin{array}{l}5.00 \\
2.66\end{array}$ & $\begin{array}{l}6.00 \\
2.04\end{array}$ & $\begin{array}{l}7.00 \\
1.55\end{array}$ & $\begin{array}{l}8.00 \\
1,15\end{array}$ & $\begin{array}{l}9.00 \\
0.82\end{array}$ & $\begin{array}{r}10.00 \\
0.56\end{array}$ & $\begin{array}{r}11.00 \\
0.36\end{array}$ & $\begin{array}{r}12.00 \\
0.21\end{array}$ & $\begin{array}{r}13.00 \\
0.10\end{array}$ & $\begin{array}{r}14.00 \\
0.03\end{array}$ & 15.00 & 16.00 & 9.85 & 7.28 \\
\hline 38 & & 2 & 1.00 & $\begin{array}{l}2.00 \\
7.96\end{array}$ & $\begin{array}{l}3.00 \\
6.19\end{array}$ & $\begin{array}{l}4.00 \\
4.90\end{array}$ & $\begin{array}{l}5.00 \\
3.88\end{array}$ & $\begin{array}{l}6.00 \\
3.07\end{array}$ & $\begin{array}{l}7.00 \\
2.41\end{array}$ & $\begin{array}{l}8.00 \\
1.87\end{array}$ & $\begin{array}{l}9.00 \\
1.42\end{array}$ & $\begin{array}{r}10.00 \\
1.06\end{array}$ & $\begin{array}{r}11.00 \\
0.76\end{array}$ & $\begin{array}{r}12.00 \\
0.52\end{array}$ & $\begin{array}{r}13.00 \\
0.33\end{array}$ & $\begin{array}{r}14.00 \\
0.19\end{array}$ & $\begin{array}{r}15.00 \\
0.09\end{array}$ & $\begin{array}{r}16.00 \\
0.03\end{array}$ & 17.00 & 10.64 \\
\hline 40 & & 2 & 1.00 & $\begin{array}{r}2,00 \\
11.43\end{array}$ & $\begin{array}{l}3.00 \\
8.64\end{array}$ & $\begin{array}{l}4.00 \\
6.80\end{array}$ & $\begin{array}{l}5.00 \\
5.44\end{array}$ & $\begin{array}{l}6.00 \\
4.36\end{array}$ & $\begin{array}{l}7.00 \\
3.50\end{array}$ & $\begin{array}{l}8.00 \\
2.79\end{array}$ & $\begin{array}{l}9.00 \\
2.21\end{array}$ & $\begin{array}{r}10.00 \\
1.72\end{array}$ & $\begin{array}{r}11.00 \\
1.32\end{array}$ & $\begin{array}{r}12.00 \\
0.98\end{array}$ & $\begin{array}{l}13.00 \\
0.71\end{array}$ & $\begin{array}{r}14.00 \\
0.49\end{array}$ & $\begin{array}{r}15.00 \\
0.31\end{array}$ & $\begin{array}{r}16.00 \\
0.18\end{array}$ & $\begin{array}{r}17.00 \\
0.09\end{array}$ & $\begin{array}{r}18.00 \\
0.03\end{array}$ \\
\hline
\end{tabular}


Tabla A.2

Coeficientes de la función de distribución del cuadrado de la máxima correlación canónica muestral en el caso nulo, $F(x ; p, q, n)=x^{b}\left(a_{0}+a_{1}(1-x)+\ldots+a_{k}(1-x)^{k}\right)$ para $d=p+q=5$

\begin{tabular}{|c|c|c|c|c|c|c|c|c|c|c|c|c|c|c|c|c|c|c|c|}
\hline$n$ & $p$ & $b$ & $a_{0}$ & $a_{1}$ & $a_{2}$ & $a_{3}$ & $a_{4}$ & $a_{5}$ & $a_{6}$ & $a_{7}$ & $a_{8}$ & $a_{9}$ & $a_{10}$ & $a_{11}$ & $a_{12}$ & $a_{13}$ & $a_{14}$ & $a_{15}$ & $a_{16}$ \\
\hline 7 & & 2 & 1.00 & & & & & & & & & & & & & & & & \\
\hline 9 & & 2 & 1.00 & 2.00 & & & & & & & & & & & & & & & \\
\hline 11 & & 2 & 1.00 & 2.00 & 3.00 & & & & & & & & & & & & & & \\
\hline 13 & & 2 & 1.00 & 2.00 & 3.00 & 4.00 & & & & & & & & & & & & & \\
\hline 15 & & 2 & 1.00 & 2.00 & 3.00 & 4.00 & 5.00 & & & & & & & & & & & & \\
\hline 17 & & 2 & 1,00 & 2.00 & 3.00 & 4.00 & 5.00 & 6.00 & & & & & & & & & - & & \\
\hline 19 & & 2 & 1.00 & 2.00 & 3.00 & 4.00 & 5.00 & 6.00 & 7.00 & & & & & & & & & & \\
\hline 21 & & 2 & 1.00 & 2.00 & 3.00 & 4.00 & 5.00 & 6.00 & 7.00 & 8.00 & & & & & & & & & \\
\hline 23 & 1 & 2 & 1.00 & 2.00 & 3.00 & 4.00 & 5.00 & 6.00 & 7.00 & 8.00 & 9.00 & & & & & & & & \\
\hline 25 & & 2 & 1.00 & 2.00 & 3.00 & 4.00 & 5.00 & 6.00 & 7.00 & 8.00 & 9.00 & 10.00 & & & & & & & \\
\hline 27 & & 2 & 1.00 & 2.00 & 3.00 & 4.00 & 5.00 & 6.00 & 7.00 & 8.00 & 9.00 & 10.00 & 11.00 & & & & & & \\
\hline 29 & & 2 & 1.00 & 2.00 & 3.00 & 4.00 & 5.00 & 6.00 & 7.00 & 8.00 & 9.00 & 10.00 & 11.00 & 12.00 & & & & & \\
\hline 31 & & 2 & 1.00 & 2.00 & 3.00 & 4.00 & 5.00 & 6.00 & 7.00 & 8.00 & 9.00 & 10.00 & 11.00 & 12.00 & 13.00 & & & & \\
\hline 33 & & 2 & 1.00 & 2.00 & 3.00 & 4.00 & 5.00 & 6.00 & 7.00 & 8.00 & 9.00 & 10.00 & 11.00 & 12.00 & 13.00 & 14.00 & & & \\
\hline 35 & & 2 & 1.00 & 2.00 & 3.00 & 4.00 & 5.00 & 6.00 & 7.00 & 8.00 & 9.00 & 10.00 & 11.00 & 12.00 & 13.00 & 14.00 & 15.00 & & \\
\hline 37 & & 2 & 1.00 & 2.00 & 3.00 & 4.00 & 5.00 & 6.00 & 7.00 & 8.00 & 9.00 & 10.00 & 11.00 & 12.00 & 13.00 & 14.00 & 15.00 & 16.00 & \\
\hline 39 & & 2 & 1.00 & 2.00 & 3.00 & 4.00 & 5.00 & 6.00 & 7.00 & 8.00 & 9.00 & 10.00 & 11.00 & 12.00 & 13.00 & 14.00 & 15.00 & 16.00 & 17.00 \\
\hline
\end{tabular}




\section{Tabla A.2}

(Continuación)

\begin{tabular}{|c|c|c|c|c|c|c|c|c|c|c|c|c|c|c|c|c|c|c|c|}
\hline$n$ & $p$ & $b$ & $a_{0}$ & $\begin{array}{c}a_{1} \\
a_{17}\end{array}$ & $\begin{array}{c}a_{2} \\
a_{18}\end{array}$ & $\begin{array}{c}a_{3} \\
a_{19}\end{array}$ & $\begin{array}{c}a_{4} \\
a_{20}\end{array}$ & $\begin{array}{c}a_{5} \\
a_{21}\end{array}$ & $\begin{array}{c}a_{6} \\
a_{22}\end{array}$ & $\begin{array}{c}a_{7} \\
a_{23}\end{array}$ & $\begin{array}{c}a_{8} \\
a_{24}\end{array}$ & $\begin{array}{c}a_{9} \\
a_{25}\end{array}$ & $\begin{array}{l}a_{10} \\
a_{26}\end{array}$ & $\begin{array}{l}a_{11} \\
a_{27}\end{array}$ & $\begin{array}{l}a_{12} \\
a_{28}\end{array}$ & $\begin{array}{l}a_{13} \\
a_{29}\end{array}$ & $\begin{array}{l}a_{14} \\
a_{30}\end{array}$ & $\begin{array}{l}a_{15} \\
a_{31}\end{array}$ & $\begin{array}{l}a_{16} \\
a_{32}\end{array}$ \\
\hline 7 & & 3 & 1.00 & & & & & & & & & & & & & & & & \\
\hline 9 & & 3 & 1.00 & 3.00 & 1.00 & & & & & & & & & & & & & & \\
\hline 11 & & 3 & 1.00 & 3.00 & 6.00 & 3.00 & 1.00 & & & & & & & & & & & & \\
\hline 13 & & 3 & 1.00 & 3.00 & 6.00 & 10.00 & 6.00 & 3.00 & 1.00 & & & & & & & & & & \\
\hline $15^{\circ}$ & & 3 & 1.00 & 3.00 & 6.00 & 10.00 & 15.00 & 10.00 & 6.00 & 3.00 & 1.00 & & & & & & & & \\
\hline 17 & & 3 & 1.00 & 3.00 & 6.00 & 10.00 & 15.00 & 21.00 & 15.00 & 10.00 & 6.00 & 3,00 & 1.00 & & & & & & \\
\hline 19 & & 3 & 1.00 & 3.00 & 6.00 & 10.00 & 15.00 & 21.00 & 28.00 & 21.00 & 15.00 & 10.00 & 6.00 & 3.00 & 1,00 & & & & \\
\hline 21 & & 3 & 1.00 & 3.00 & 6.00 & 10.00 & 15.00 & 21.00 & 28.00 & 36.00 & 28.00 & 21.00 & 15.00 & 10.00 & 6.00 & 3.00 & 1.00 & & \\
\hline 23 & & 3 & 1.00 & 3.00 & 6.00 & 10.00 & 15.00 & 21.00 & 28.00 & 36.00 & 45.00 & 36.00 & 28.00 & 21.00 & 15.00 & 10.00 & 6.00 & 3.00 & 1.00 \\
\hline 25 & & 3 & 1.00 & $\begin{array}{l}3.00 \\
3.00\end{array}$ & $\begin{array}{l}6.00 \\
1.00\end{array}$ & 10.00 & 15.00 & 21.00 & 28.00 & 36.00 & 45.00 & 55.00 & 45.00 & 36.00 & 28.00 & 21.00 & 15.00 & 10.00 & 6.00 \\
\hline 27 & 2 & 3 & 1.00 & $\begin{array}{r}3.00 \\
10.00\end{array}$ & $\begin{array}{l}6.00 \\
6.00\end{array}$ & $\begin{array}{c}10.00 \\
3.00\end{array}$ & $\begin{array}{r}15.00 \\
1.00\end{array}$ & 21.00 & 28.00 & 36.00 & 45.00 & 55.00 & 66.00 & 55.00 & 45.00 & 36.00 & 28.00 & 21.00 & 15.00 \\
\hline 29 & & 3 & 1.00 & $\begin{array}{r}3.00 \\
21.00\end{array}$ & $\begin{array}{r}6.00 \\
15.00\end{array}$ & $\begin{array}{l}10.00 \\
10.00\end{array}$ & $\begin{array}{r}15.00 \\
6.00\end{array}$ & $\begin{array}{r}21.00 \\
3.00\end{array}$ & $\begin{array}{r}28.00 \\
1.00\end{array}$ & 36.00 & 45.00 & 55.00 & 66,00 & 78.00 & 66.00 & 55.00 & 45.00 & 36.00 & 28.00 \\
\hline 31 & & 3 & 1.00 & $\begin{array}{r}3.00 \\
36.00\end{array}$ & $\begin{array}{r}6.00 \\
28.00\end{array}$ & $\begin{array}{l}10.00 \\
21.00\end{array}$ & $\begin{array}{l}15.00 \\
15.00\end{array}$ & $\begin{array}{l}21.00 \\
10.00\end{array}$ & $\begin{array}{r}28.00 \\
6.00\end{array}$ & $\begin{array}{r}36.00 \\
3.00\end{array}$ & $\begin{array}{r}45.00 \\
1.00\end{array}$ & 55.00 & 66.00 & 78.00 & 91.00 & 78.00 & 66.00 & 55.00 & 45.00 \\
\hline 33 & & 3 & 1.00 & $\begin{array}{r}3.00 \\
55.00\end{array}$ & $\begin{array}{r}6.00 \\
45.00\end{array}$ & $\begin{array}{l}10.00 \\
36.00\end{array}$ & $\begin{array}{l}15.00 \\
28.00\end{array}$ & $\begin{array}{l}21.00 \\
21.00\end{array}$ & $\begin{array}{l}28.00 \\
15.00\end{array}$ & $\begin{array}{l}36.00 \\
10.00\end{array}$ & $\begin{array}{r}45.00 \\
6.00\end{array}$ & $\begin{array}{r}55.00 \\
3.00\end{array}$ & $\begin{array}{r}66.00 \\
1.00\end{array}$ & 78.00 & 91.00 & 105.00 & 91.00 & 78.00 & 66.00 \\
\hline 35 & & 3 & 1.00 & $\begin{array}{r}3.00 \\
78.00\end{array}$ & $\begin{array}{r}6.00 \\
66.00\end{array}$ & $\begin{array}{l}10.00 \\
55.00\end{array}$ & $\begin{array}{l}15.00 \\
45.00\end{array}$ & $\begin{array}{l}21.00 \\
36.00\end{array}$ & $\begin{array}{l}28.00 \\
28.00\end{array}$ & $\begin{array}{l}36.00 \\
21.00\end{array}$ & $\begin{array}{l}45.00 \\
15.00\end{array}$ & $\begin{array}{l}55.00 \\
10.00\end{array}$ & $\begin{array}{r}66.00 \\
6.00\end{array}$ & $\begin{array}{r}78.00 \\
3.00\end{array}$ & $\begin{array}{r}91.00 \\
1.00\end{array}$ & 105.00 & 120.00 & 105.00 & 91.00 \\
\hline 37 & & 3 & 1.00 & $\begin{array}{r}3.00 \\
105.00\end{array}$ & $\begin{array}{r}6.00 \\
91.00\end{array}$ & $\begin{array}{l}10.00 \\
78.00\end{array}$ & $\begin{array}{l}15.00 \\
66.00\end{array}$ & $\begin{array}{l}21.00 \\
55.00\end{array}$ & $\begin{array}{l}28.00 \\
45.00\end{array}$ & $\begin{array}{l}36.00 \\
36.00\end{array}$ & $\begin{array}{l}45.00 \\
28.00\end{array}$ & $\begin{array}{l}55.00 \\
21.00\end{array}$ & $\begin{array}{l}66.00 \\
15.00\end{array}$ & $\begin{array}{l}78.00 \\
10.00\end{array}$ & $\begin{array}{r}91.00 \\
6.00\end{array}$ & $\begin{array}{r}105.00 \\
3.00\end{array}$ & $\begin{array}{r}120.00 \\
1.00\end{array}$ & 136.00 & 120.00 \\
\hline 39 & & 3 & 1.00 & $\begin{array}{r}3.00 \\
136.00\end{array}$ & $\begin{array}{r}6.00 \\
120.00\end{array}$ & $\begin{array}{r}10.00 \\
105.00\end{array}$ & $\begin{array}{l}15.00 \\
91.00\end{array}$ & $\begin{array}{l}21.00 \\
78.00\end{array}$ & $\begin{array}{l}28.00 \\
66.00\end{array}$ & $\begin{array}{l}36.00 \\
55.00\end{array}$ & $\begin{array}{l}45.00 \\
45.00\end{array}$ & $\begin{array}{l}55.00 \\
36.00\end{array}$ & $\begin{array}{l}66.00 \\
28.00\end{array}$ & $\begin{array}{l}78.00 \\
21.00\end{array}$ & $\begin{array}{l}91.00 \\
15.00\end{array}$ & $\begin{array}{c}105.00 \\
10.00\end{array}$ & $\begin{array}{r}120.00 \\
6.00\end{array}$ & $\begin{array}{r}136.00 \\
3.00\end{array}$ & $\begin{array}{r}153.00 \\
1.00\end{array}$ \\
\hline
\end{tabular}




\section{Tabla A.3}

Coeficientes de la función de distribución del cuadrado de la máxima correlación canónica muestral en el caso nulo. $F(x ; p, q, n)=x^{b}\left(a_{0}+a_{1}(1-x)+\ldots+a_{k}(1-x)^{k}\right)$ para $d=p+q=6$

\begin{tabular}{|c|c|c|c|c|c|c|c|c|c|c|c|c|c|c|c|c|c|c|c|}
\hline$n$ & $p$ & $b$ & $a_{0}$ & $a_{1}$ & $a_{2}$ & $a_{3}$ & $a_{4}$ & $a_{5}$ & $a_{6}$ & $a_{7}$ & $a_{8}$ & $a_{9}$ & $a_{10}$ & $a_{11}$ & $a_{12}$ & $a_{13}$ & $a_{14}$ & $a_{15}$ & $a_{16}$ \\
\hline 8 & & $7 / 2$ & 1.00 & & & & & & & & & & & & & & & & \\
\hline 10 & & $7 / 2$ & 1.00 & 2.50 & & & & & & & & & & & & & & & \\
\hline 12 & & $7 / 2$ & 1.00 & 2.50 & 4.38 & & & & & & & & & & & & & & \\
\hline 14 & & $7 / 2$ & 1,00 & 2.50 & 4.38 & 6.56 & & & & & & & & & & & & & \\
\hline 16 & & $7 / 2$ & 1.00 & 2.50 & 4.38 & 6.56 & 9.02 & & & & & & . & & & & & & \\
\hline 18 & & $7 / 2$ & 1.00 & 2.50 & 4.38 & 6.56 & 9.02 & 11.73 & & & & & & & & & & & \\
\hline 20 & & $7 / 2$ & 1.00 & 2.50 & 4.38 & 6.56 & 9.02 & 11.73 & 14.66 & & & & & & & & & & \\
\hline 22 & & $7 / 2$ & 1.00 & 2.50 & 4.38 & 6.56 & 9.02 & 11.73 & 14.66 & 17,80 & & & & & & & & & \\
\hline 24 & 1 & $7 / 2$ & 1.00 & 2.50 & 4.38 & 6.56 & 9.02 & 11.73 & 14.66 & 17.80 & 21.14 & & & & & & & & \\
\hline 26 & & $7 / 2$ & 1.00 & 2.50 & 4.38 & 6.56 & 9.02 & 11.73 & 14.66 & 17.80 & 21.14 & 24.67 & & & & & & & \\
\hline 28 & & $7 / 2$ & 1.00 & 2.50 & 4.38 & 6.56 & 9.02 & 11.73 & 14.66 & 17.80 & 21.14 & 24.67 & 28.37 & & & & & & \\
\hline 30 & & $7 / 2$ & 1.00 & 2.50 & 4.38 & 6.56 & 9.02 & 11.73 & 14.66 & 17.80 & 21.14 & 24.67 & 28.37 & 32.24 & & & & & \\
\hline 32 & & $7 / 2$ & 1.00 & 2.50 & 4.38 & 6.56 & 9.02 & 11.73 & 14.66 & 17.80 & 21.14 & 24.67 & 28.37 & 32.24 & 36.27 & & & & \\
\hline 34 & & $7 / 2$ & 1.00 & 2.50 & 4.38 & 6.56 & 9.02 & 11.73 & 14.66 & 17.80 & 21.14 & 24.67 & 28.37 & 32.24 & 36.27 & 40.45 & & & \\
\hline 36 & & $7 / 2$ & 1.00 & 2.50 & 4.38 & 6.56 & 9.02 & 11.73 & 14.66 & 17.80 & 21.14 & 24.67 & 28.37 & 32.24 & 36.27 & 40.45 & 44.78 & & \\
\hline 38 & & $7 / 2$ & 1.00 & 2.50 & 4.38 & 6.56 & 9.02 & 11.73 & 14.66 & 17.80 & 21.14 & 24.67 & 28.37 & 32.24 & 36.27 & 40.45 & 44.78 & 49.26 & \\
\hline 40 & & $7 / 2$ & 1.00 & 2.50 & 4.38 & 6.56 & 9.02 & 11.73 & 14.66 & 17.80 & 21.14 & 24.67 & 28.37 & 32.24 & 36.27 & 40.45 & 44.78 & 49.26 & 53. \\
\hline
\end{tabular}


Tabla A.3

(Continuación)

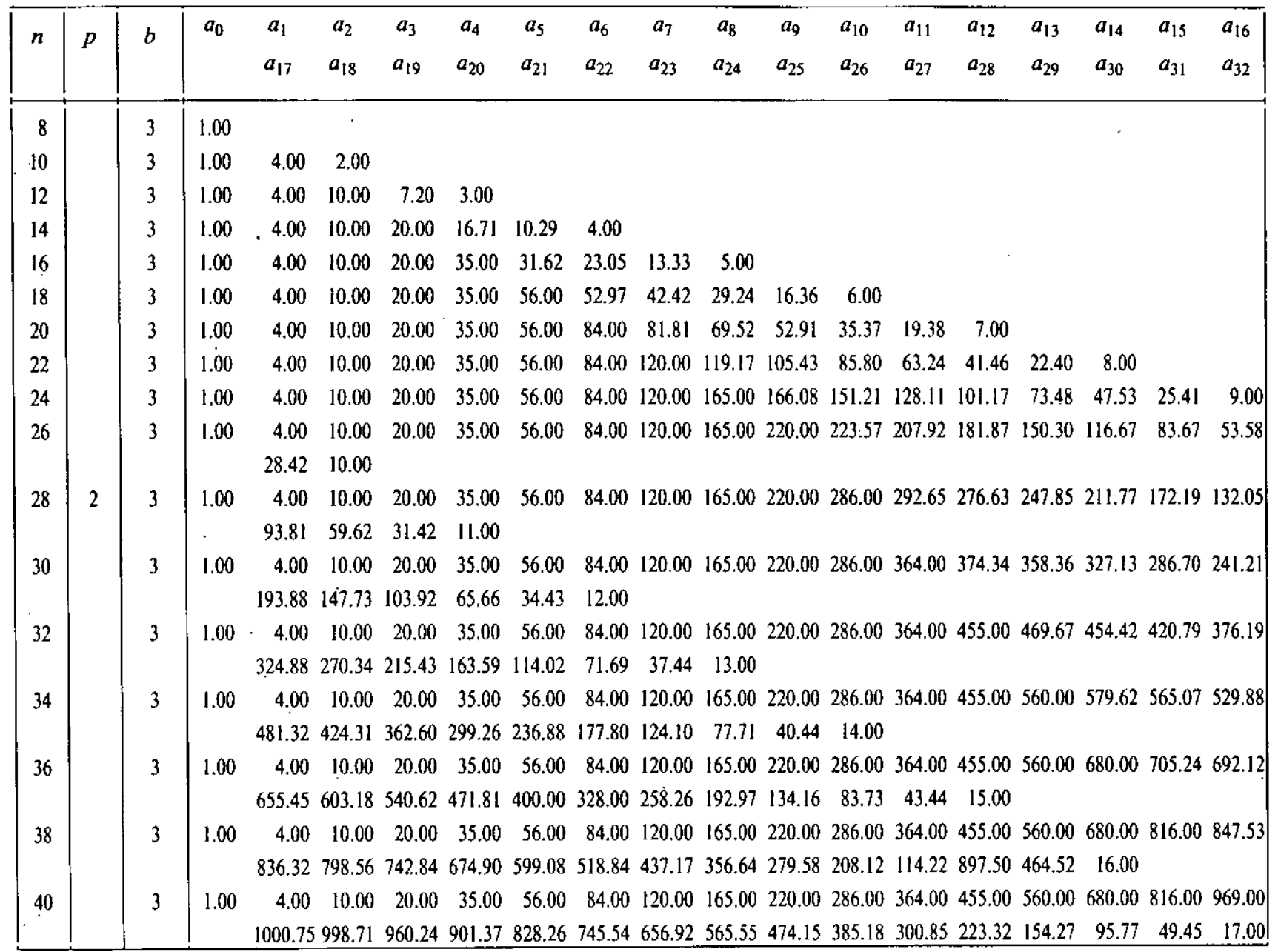


Tabla A.3

\section{(Continuación)}

\begin{tabular}{|c|c|c|c|c|c|c|c|c|c|c|c|c|c|c|c|c|c|}
\hline$n$ & $p$ & $b$ & $a_{0}$ & $\begin{array}{c}a_{1} \\
a_{15}\end{array}$ & $\begin{array}{c}a_{2} \\
a_{16}\end{array}$ & $\begin{array}{c}a_{3} \\
a_{17}\end{array}$ & $\begin{array}{c}a_{4} \\
a_{18}\end{array}$ & $\begin{array}{c}a_{5} \\
a_{19}\end{array}$ & $\begin{array}{c}a_{6} \\
a_{20}\end{array}$ & $\begin{array}{l}a_{7} \\
a_{21}\end{array}$ & $\begin{array}{c}a_{8} \\
a_{22}\end{array}$ & $\begin{array}{c}a_{9} \\
a_{23}\end{array}$ & $\begin{array}{l}a_{10} \\
a_{24}\end{array}$ & $\begin{array}{l}a_{11} \\
a_{25}\end{array}$ & $\begin{array}{l}a_{12} \\
a_{26}\end{array}$ & $\begin{array}{l}a_{13} \\
a_{27}\end{array}$ & $a_{14}$ \\
\hline 8 & \multirow{15}{*}{3} & $9 / 2$ & 1.00 & & & & & & & & & & & & & & \\
\hline 10 & & $9 / 2$ & 1.00 & 4.50 & 3.00 & 0.25 & & & & & & & & & & & \\
\hline 12 & & $9 / 2$ & 1.00 & 4.50 & 12.38 & 11.50 & 6.25 & 1.00 & 0.13 & & & & & & & & \\
\hline 14 & & $9 / 2$ & 1.00 & 4.50 & 12.33 & 26.81 & 28.13 & 21.23 & 11.06 & 2.53 & 0.56 & 0.08 & & & & & \\
\hline 16 & & $9 / 2$ & 1.00 & 4.50 & 12.38 & 26.81 & 50.27 & 55.69 & 48.47 & 34.03 & 17.79 & 5.16 & 1.55 & 0.38 & 0.06 & & \\
\hline 18 & & $9 / 2$ & 1.00 & 4.50 & 12.38 & 26.81 & 50.27 & 85.46 & 97.20 & 91.33 & 73.73 & 50.27 & 26.81 & 9.22 & 3.35 & 1.08 & 0.27 \\
\hline & & & & 0.04 & & & & & & & & & & & & & \\
\hline \multirow[t]{2}{*}{20} & & $9 / 2$ & 1.00 & 4.50 & 12.38 & 26.81 & 50.27 & 85.46 & 135.32 & 155.85 & 153.33 & 134.06 & 104.32 & 70.38 & 38.54 & 15.08 & 6.28 \\
\hline & & & & 2.43 & 0.81 & 0.21 & 0.03 & & & & & & & & & & \\
\hline \multirow[t]{2}{*}{22} & & $9 / 2$ & 1.00 & 4.50 & 12.38 & 26.81 & 50.27 & 85.46 & I 35.32 & 202.98 & 235.03 & 238.14 & 219.60 & 184.28 & 140.66 & 94.81 & 53.41 \\
\hline & & & & 23.15 & 10.68 & 4.70 & 1.88 & 0.64 & 0.17 & 0.03 & & & & & & & \\
\hline \multirow[t]{2}{*}{24} & & $9 / 2$ & 1.00 & 4.50 & 12.38 & 26.81 & 50.27 & 85.46 & 135.32 & 202.98 & 291.78 & 338.30 & 349.58 & 332.66 & 294.60 & 242.45 & 183.25 \\
\hline & & & & 121.04 & 71.89 & 33.83 & 16.91 & 81.92 & 3.71 & 1.51 & 0.52 & 0.14 & 0.02 & & & & \\
\hline \multirow[t]{2}{*}{26} & & $9 / 2$ & 1.00 & 4.50 & 12.38 & 26.81 & 50.27 & 85.46 & 135.32 & 202.98 & 291.78 & 405.24 & 469.39 & 491.59 & 479.26 & 439.79 & 380.59 \\
\hline & & & & 309.05 & 232.58 & 158.58 & 94.44 & 47.57 & 25.37 & 13.24 & 6.57 & 3.03 & 1.25 & 0.44 & 0.12 & 0.02 & \\
\hline
\end{tabular}


Tabla A.4

Coeficientes de la función de distribución del cuadrado de la máxima correlación canónica muestral en el caso nulo,

$F(x ; p, q, n)=x^{b}\left(a_{0}+a_{1}(1-x)+\ldots+a_{k}(1-x)^{k}\right)$ para $d=p+q=7$

\begin{tabular}{|c|c|c|c|c|c|c|c|c|c|c|c|c|c|c|c|c|c|c|}
\hline$n$ & $p$ & $b$ & $a_{0}$ & $a_{1}$ & $a_{2}$ & $a_{3}$ & $a_{4}$ & $a_{5}$ & $a_{6}$ & $a_{7}$ & $a_{8}$ & $a_{9}$ & $a_{10}$ & $a_{11}$ & $a_{12}$ & $a_{13}$ & $a_{14}$ & $a_{15}$ \\
\hline 9 & & 3 & 1.00 & & & & & & & & & & & & & & & \\
\hline 11 & & 3 & 1.00 & 3.00 & & & & & & & & & & & & & & \\
\hline 13 & & 3 & 1.00 & 3.00 & 6.00 & & & & & & & & & & & & & \\
\hline 15 & & 3 & 1.00 & 3.00 & 6.00 & 10.00 & & & & & & & & & & & & \\
\hline 17 & & 3 & 1.00 & 3.00 & 6.00 & 10.00 & 15.00 & & & & & & & & & & & \\
\hline 19 & & 3 & 1.00 & 3.00 & 6.00 & 10.00 & 15.00 & 21.00 & & & & & & & & & & \\
\hline 21 & & 3 & 1.00 & 3.00 & 6.00 & 10.00 & 15.00 & $21: 00$ & 28.00 & & & & & & & & & \\
\hline 23 & 1 & 3 & 1.00 & 3.00 & 6.00 & 10.00 & 15.00 & 21.00 & 28.00 & 36.00 & & & & & & & & \\
\hline 25 & & 3 & 1.00 & 3.00 & 6.00 & 10.00 & 15.00 & 21.00 & 28.00 & 36.00 & 45.00 & . & & & & & & \\
\hline 27 & & 3 & 1.00 & 3.00 & 6.00 & 10.00 & 15.00 & 21.00 & 28.00 & 36.00 & 45.00 & 55.00 & & & & & & \\
\hline 29 & & 3 & 1.00 & 3.00 & 6.00 & 10.00 & 15.00 & 21.00 & 28.00 & 36.00 & 45.00 & 55.00 & 66.00 & & & & & \\
\hline 31 & & 3 & 1.00 & 3.00 & 6.00 & 10.00 & 15.00 & 21.00 & 28.00 & 36.00 & 45.00 & 55.00 & 66.00 & 78.00 & & & & \\
\hline 33 & & 3 & 1.00 & 3.00 & 6.00 & 10.00 & 15.00 & 21.00 & 28.00 & 36.00 & 45.00 & 55.00 & 66.00 & 78.00 & 91.00 & & & \\
\hline 35 & & 3 & 1.00 & 3.00 & 6.00 & 10.00 & 15.00 & 21.00 & 28.00 & 36.00 & 45.00 & 55.00 & 66.00 & 78.00 & 91.00 & 105.00 & & \\
\hline 37 & & 3 & 1.00 & 3.00 & 6.00 & 10.00 & 15.00 & 21.00 & 28.00 & 36.00 & 45.00 & 55.00 & 66.00 & 78.00 & 91.00 & 105.00 & 120.00 & \\
\hline 39 & & 3 & 1.00 & 3.00 & 6.00 & 10.00 & 15.00 & 21.00 & 28.00 & 36.00 & 45.00 & 55.00 & 66.00 & 78.00 & 91.00 & 105.00 & 120.00 & 136.00 \\
\hline
\end{tabular}


Tabla A.4

(Continuación)

\begin{tabular}{|c|c|c|c|c|c|c|c|c|c|c|c|c|c|c|c|c|c|c|}
\hline$n$ & $p$ & $b$ & $a_{0}$ & $\begin{array}{l}a_{1} \\
a_{16}\end{array}$ & $\begin{array}{l}a_{2} \\
a_{17}\end{array}$ & $\begin{array}{l}a_{3} \\
a_{18}\end{array}$ & $\begin{array}{l}a_{4} \\
a_{19}\end{array}$ & $\begin{array}{l}a_{5} \\
a_{20}\end{array}$ & $\begin{array}{l}a_{6} \\
a_{21}\end{array}$ & $\begin{array}{l}a_{7} \\
a_{22}\end{array}$ & $\begin{array}{l}a_{8} \\
a_{23}\end{array}$ & $\begin{array}{l}a_{9} \\
a_{24}\end{array}$ & $\begin{array}{l}a_{10} \\
a_{25}\end{array}$ & $\begin{array}{l}a_{11} \\
a_{26}\end{array}$ & $\begin{array}{l}a_{12} \\
a_{27}\end{array}$ & $\begin{array}{l}a_{13} \\
a_{28}\end{array}$ & $\begin{array}{l}a_{14} \\
a_{29}\end{array}$ & $\begin{array}{l}a_{15} \\
a_{30}\end{array}$ \\
\hline 9 & & 5 & 1.00 & & & & & & & & & & & & & & & \\
\hline $1 !$ & & 5 & 1.00 & 5.00 & 3.73 & & & & & & & & & & & & & \\
\hline 13 & & 5 & 1.00 & 5.00 & 15.00 & 14.00 & 7.00 & & & & & & & & & . & . & \\
\hline 15 & & 5 & 1.00 & 5.00 & 15.00 & 35.00 & 37.00 & 27.00 & 12,00 & & & & & & & & & \\
\hline 17 & & 5 & 1.00 & 5.00 & 15.00 & 35.00 & 70.00 & 78.33 & 67.00 & 44.00 & 18.33 & & & & & & & \\
\hline 19 & & 5 & 1.00 & 5.00 & 15,00 & 35.00 & 70.00 & 126.00 & 145.00 & 135.00 & 105.00 & 65.00 & 26.00 & & & & & \\
\hline 21 & & 5 & 1.00 & 5.00 & 15.00 & 35.00 & 70.00 & 126.00 & 210.00 & 245.00 & 240.00 & 205.00 & 151.00 & 90.00 & 35.00 & & & \\
\hline 23 & & 5 & 1.00 & 5.00 & 15.00 & 35.00 & 70.00 & 126.00 & 210.00 & 330.00 & 387.33 & 392.00 & 355.00 & 288.33 & 205.00 & $0 \quad 119.00$ & 45.33 & \\
\hline 25 & & 5 & 1.00 & $\begin{array}{r}5.00 \\
57.00\end{array}$ & 15.00 & 35.00 & 70.00 & 126.00 & 210.00 & 330.00 & 495.00 & 582,00 & 602.00 & 567.00 & 490.00 & $0 \quad 385.00$ & 267,00 & 152.00 \\
\hline 27 & 2 & 5 & 1.00 & $\begin{array}{r}5.00 \\
337.00\end{array}$ & $\begin{array}{r}15.00 \\
189.00\end{array}$ & $\begin{array}{l}35.00 \\
70.00\end{array}$ & 70.00 & 126.00 & 210.00 & 330.00 & 495.00 & 715.00 & 840.00 & 882.00 & 854.00 & $0 \quad 770.00$ & 645.00 & 495.00 \\
\hline 29 & & 5 & 1.00 & $\begin{array}{r}5.00 \\
820.00\end{array}$ & $\begin{array}{r}15.00 \\
618.33\end{array}$ & $\begin{array}{r}35.00 \\
415.00\end{array}$ & $\begin{array}{r}70.00 \\
230.00\end{array}$ & $\begin{array}{r}126.00 \\
84.33\end{array}$ & 210.00 & 330.00 & 495.00 & 715.00 & $100 ! .00$ & 1173.33 & 1245.00 & 01230.00 & 1143.00 & 1001.00 \\
\hline 31 & & 5 & $1,00^{\circ}$ & $\begin{array}{r}5.00 \\
1470.00\end{array}$ & $\begin{array}{r}15.00 \\
1260.00\end{array}$ & $\begin{array}{r}35.00 \\
1015.00\end{array}$ & $\begin{array}{r}70.00 \\
755.00\end{array}$ & $\begin{array}{l}126.00 \\
501.00\end{array}$ & $\begin{array}{l}210.00 \\
275.00\end{array}$ & $\begin{array}{l}330.00 \\
100.00\end{array}$ & 495.00 & 715.00 & 1001.00 & 1365.00 & 1595.00 & 01705.00 & 1710.00 & 1626.00 \\
\hline 33 & & 5 & 1.00 & $\begin{array}{r}5.00 \\
2235.00\end{array}$ & $\begin{array}{r}15.00 \\
2070.00\end{array}$ & $\begin{array}{r}35.00 \\
1834.00\end{array}$ & $\begin{array}{r}70.00 \\
1547.00\end{array}$ & $\begin{array}{r}126.00 \\
1230.00\end{array}$ & $\begin{array}{l}210.00 \\
905.00\end{array}$ & $\begin{array}{l}330.00 \\
595.00\end{array}$ & $\begin{array}{l}495.00 \\
324.00\end{array}$ & $\begin{array}{l}715.00 \\
117.00\end{array}$ & 1001.00 & 1365.00 & 1820.00 & 02119.00 & 2277.00 & 2310.00 \\
\hline 35 & & 5 & 1.00 & $\begin{array}{r}5,00 \\
3047.00\end{array}$ & $\begin{array}{r}15.00 \\
2988.33\end{array}$ & $\begin{array}{r}35.00 \\
2820.00\end{array}$ & $\begin{array}{r}70.00 \\
2562.00\end{array}$ & $\begin{array}{r}126.00 \\
2235.33\end{array}$ & $\begin{array}{r}210.00 \\
1862.00\end{array}$ & $\begin{array}{r}330.00 \\
1465.00\end{array}$ & $\begin{array}{r}495.00 \\
1068.33\end{array}$ & $\begin{array}{r}715.00 \\
697.00\end{array}$ & $\begin{array}{l}1001.00 \\
377.00\end{array}$ & $\begin{array}{r}1365.00 \\
135.33\end{array}$ & 1820.00 & 2380.00 & 2760.33 & 2977.00 \\
\hline 37 & & 5 & 1.00 & $\begin{array}{r}5.00 \\
3822.00\end{array}$ & $\begin{array}{r}15.00 \\
3939.00\end{array}$ & $\begin{array}{r}35.00 \\
3905.00\end{array}$ & $\begin{array}{r}70.00 \\
3740.00\end{array}$ & $\begin{array}{r}126.00 \\
3465.00\end{array}$ & $\begin{array}{r}210.00 \\
3102.00\end{array}$ & $\begin{array}{r}330.00 \\
2674.00\end{array}$ & $\begin{array}{r}495.00 \\
2205.00\end{array}$ & $\begin{array}{r}715.00 \\
1720.00\end{array}$ & $\begin{array}{l}1001.00 \\
1245.00\end{array}$ & $\begin{array}{r}1365.00 \\
807.00\end{array}$ & $\begin{array}{r}1820.00 \\
434.00\end{array}$ & $\begin{array}{r}2380.00 \\
155.00\end{array}$ & 3060.00 & 3535.00 \\
\hline 39 & & 5 & 1.00 & $\begin{array}{r}5.00 \\
4460.00\end{array}$ & $\begin{array}{r}15.00 \\
4830.00\end{array}$ & $\begin{array}{r}35.00 \\
5005.00\end{array}$ & $\begin{array}{r}70.00 \\
5005.00\end{array}$ & $\begin{array}{r}126.00 \\
4851.00\end{array}$ & $\begin{array}{r}210.00 \\
4565.00\end{array}$ & $\begin{array}{r}330.00 \\
4170.00\end{array}$ & $\begin{array}{r}495.00 \\
3690.00\end{array}$ & $\begin{array}{r}715.00 \\
3150.00\end{array}$ & $\begin{array}{l}1001.00 \\
2576.00\end{array}$ & $\begin{array}{l}1365.00 \\
1995.00\end{array}$ & $\begin{array}{l}1820.00 \\
1435.00\end{array}$ & $\begin{array}{r}2380.00 \\
925.00\end{array}$ & $\begin{array}{r}3060.00 \\
495.00\end{array}$ & $\begin{array}{r}3875.00 \\
176.00\end{array}$ \\
\hline
\end{tabular}


Tabla A.4

(Continuación)

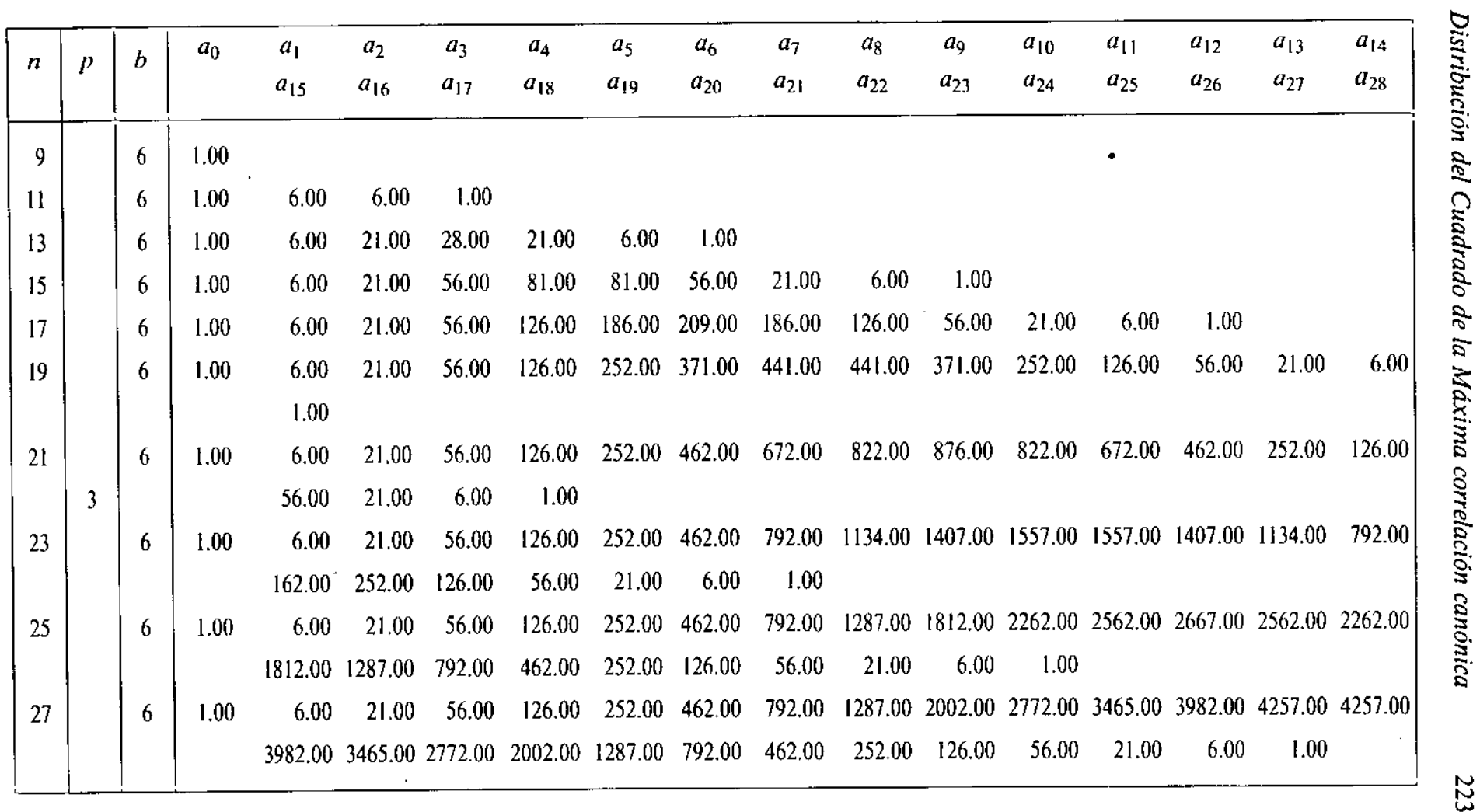


Tabla 5

Media y desviación estándar del cuadrado de la máxima correlación canónica muestral para $d=4,5,6,7$

\begin{tabular}{|c|c|c|c|c|c|c|c|c|c|c|c|c|c|c|c|c|c|c|c|c|}
\hline$d$ & & $N$ & 6 & 8 & 10 & 12 & 14 & 16 & 18 & 20 & 22 & 24 & 26 & 28 & 30 & 32 & 34 & 36 & 38 & 40 \\
\hline \multirow{4}{*}{4} & 1 & Media & 0.600 & 0.429 & 0.333 & 0.273 & 0.231 & 0.200 & 0.176 & 0.158 & 0.142 & 0 & 0.1 & 0. & 0.103 & & 0. & 86 & 0.081 & 0 \\
\hline & & $\sqrt{\text { Var }}$ & 0.263 & 0.232 & 0.200 & 0.176 & 0.155 & 0.138 & 0.122 & 0.114 & 0.105 & 0.095 & 0.089 & 0.084 & 0.077 & 0.071 & 0.071 & 0.063 & 0.063 & 0.055 \\
\hline & 2 & Media & & 0.489 & 0.385 & 0.317 & 0.269 & 0 . & 0 & & & & & & & & & & & \\
\hline & & V Var & & 24 & 97 & ( & 0.155 & 0.138 & 0.126 & 0.114 & 0.105 & 0.095 & 0.089 & 0.0 & 0.07 & 0.077 & 0.07 & 0.071 & 0.063 & 0.063 \\
\hline
\end{tabular}

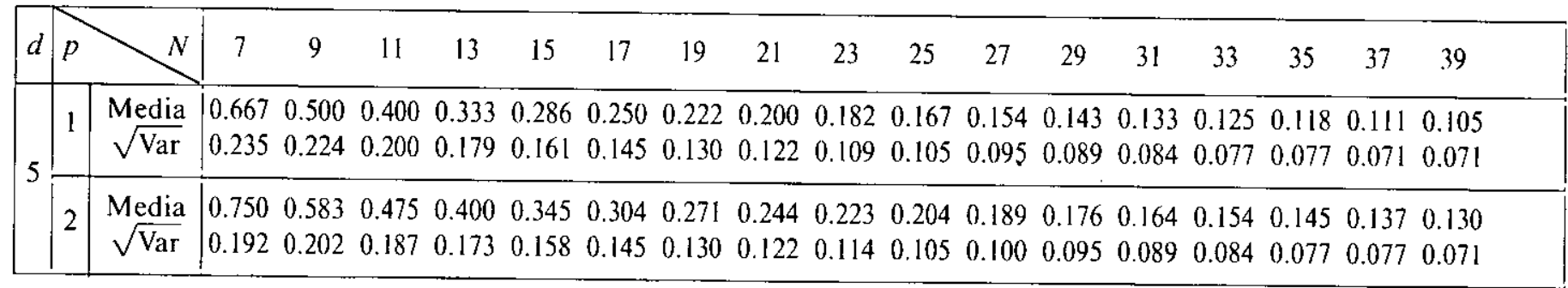

\begin{tabular}{|c|c|c|c|c|c|c|c|c|c|c|c|c|c|c|c|c|c|c|c|}
\hline$d$ & $p$ & $N$ & 8 & 10 & 12 & 14 & 16 & 18 & 20 & 22 & 24 & 26 & 28 & 30 & 32 & 34 & 36 & 38 & 40 \\
\hline \multirow{5}{*}{6} & 1 & Media & 0.714 & 0.556 & 0.455 & 0.385 & 0.333 & 0.294 & 0.263 & 0.238 & 0.217 & 0.200 & 0.185 & 0.172 & 0.161 & 0.151 & 0.143 & 0.135 & 0.128 \\
\hline & 1 & $\sqrt{\mathrm{Var}}$ & 0.212 & 0.212 & 0.195 & 0.179 & 0.161 & 0.148 & 0.134 & 0.126 & 0.118 & 0.110 & 0.100 & 0.095 & 0.089 & 0.084 & 0.084 & 0.077 & 0.071 \\
\hline & 2 & Media & 0.800 & 0.648 & 0.541 & 0.464 & 0.405 & 0.360 & 0.323 & 0.294 & 0.269 & 0.248 & 0.230 & 0.215 & 0.201 & 0.189 & 0.178 & 0.169 & 0.161 \\
\hline & 2 & $\sqrt{\operatorname{Var}}$ & 0.164 & 0.181 & 0.176 & 0.164 & 0.155 & 0.141 & 0.134 & 0.122 & 0.114 & 0.110 & 0.100 & 0.095 & 0.089 & 0.089 & 0.084 & 0.077 & 0.077 \\
\hline & 31 & Media & 0.818 & 0.670 & 0.562 & 0.483 & 0.423 & 0,377 & 0.339 & 0.308 & 0.283 & 0.261 & & & & & & & \\
\hline & 3 & $\sqrt{\operatorname{Var}}$ & 0.151 & 0.173 & 0.173 & 0.161 & 0.152 & 0.141 & 0.130 & 0.122 & 0.114 & 0.110 & & & & & & & \\
\hline
\end{tabular}


Tabla 5

(Continuación)

\begin{tabular}{|c|c|c|c|c|c|c|c|c|c|c|c|c|c|c|c|c|c|c|}
\hline$d$ & $p$ & $N$ & 9 & 11 & 13 & 15 & 17 & 19 & 21 & 23 & 25 & 27 & 29 & 31 & 33 & 35 & 37 & 39 \\
\hline & 1 & $\frac{\text { Media }}{\sqrt{\operatorname{Var}}}$ & $\begin{array}{l}0.750 \\
0.194\end{array}$ & $\begin{array}{l}0.600 \\
0.200\end{array}$ & $\begin{array}{l}0.500 \\
0.190\end{array}$ & $\begin{array}{l}0.428 \\
0.176\end{array}$ & $\begin{array}{l}0.375 \\
0.161\end{array}$ & $\begin{array}{l}0.333 \\
0.148\end{array}$ & $\begin{array}{l}0.300 \\
0.138\end{array}$ & $\begin{array}{l}0.273 \\
0.130\end{array}$ & $\begin{array}{l}0.250 \\
0.118\end{array}$ & $\begin{array}{l}0.231 \\
0.114\end{array}$ & $\begin{array}{l}0.214 \\
0.105\end{array}$ & $\begin{array}{l}0.200 \\
0.100\end{array}$ & $\begin{array}{l}0.187 \\
0.095\end{array}$ & $\begin{array}{l}0.176 \\
0.089\end{array}$ & $\begin{array}{l}0.167 \\
0.084\end{array}$ & $\begin{array}{l}0.158 \\
0.084\end{array}$ \\
\hline 7 & 2 & $\frac{\text { Media }}{\sqrt{\text { Var }}}$ & $\begin{array}{l}0.833 \\
0.141\end{array}$ & $\begin{array}{l}0.695 \\
0.164\end{array}$ & $\begin{array}{l}0.591 \\
0.164\end{array}$ & $\begin{array}{l}0.514 \\
0.158\end{array}$ & $\begin{array}{l}0.454 \\
0.148\end{array}$ & $\begin{array}{l}0.407 \\
0.141\end{array}$ & $\begin{array}{l}0.368 \\
0.130\end{array}$ & $\begin{array}{l}0.336 \\
0.122\end{array}$ & $\begin{array}{l}0.309 \\
0.118\end{array}$ & $\begin{array}{l}0.286 \\
0.110\end{array}$ & $\begin{array}{l}0.266 \\
0.105\end{array}$ & $\begin{array}{l}0.249 \\
0.095\end{array}$ & $\begin{array}{l}0.234 \\
0.095\end{array}$ & $\begin{array}{l}0.221 \\
0.089\end{array}$ & $\begin{array}{l}0.208 \\
0.084\end{array}$ & $\begin{array}{l}0.198 \\
0.084\end{array}$ \\
\hline & 3 & $\frac{\text { Media }}{\sqrt{\mathrm{Var}}}$ & $\mid \begin{array}{l}0.857 \\
0.122\end{array}$ & $\begin{array}{l}0.725 \\
0.151\end{array}$ & $\begin{array}{l}0.623 \\
0.155\end{array}$ & $\begin{array}{l}0.545 \\
0.159\end{array}$ & $\begin{array}{l}0.483 \\
0.155\end{array}$ & $\begin{array}{l}0.434 \\
0.134\end{array}$ & $\begin{array}{l}0.393 \\
0.130\end{array}$ & $\begin{array}{l}0.360 \\
0.122\end{array}$ & $\begin{array}{l}0.331 \\
0.114\end{array}$ & $\begin{array}{l}0.307 \\
0.110\end{array}$ & & & & & & \\
\hline
\end{tabular}




\section{Referencias}

Anderson, T, W. (1984). An Introduction to Mtultivariate Statistical Anallsis. Wiley and Sons, Inc., New York.

Constantini:, A. G. (1963). Some Noncentral Distribution Problems in Mullivariate Analysis, Ann. Math. Statis. 324, 1270-1285.

Constantine, A. G. (1966). The Distribution of Hotelling 's Generalised Tij, Ann. Matll. Statist. 37, 215-225.

Girsfick. M. A. (1939). On the Sampling Theory of the Roots of Determinantal Equations, Ann, Math. Statist, 10, 203-224.

Hotrilling. H. (1936). Relations Between Two Sets of Variates, Biometrika 28 , $321-337$.

Hst, L. C. (1939). On the Distribution of Roots of Certain Determinanial Equations, Ann. Eugen. Lond. 9, 250-258.

JAMl:S. A. T. (1960). The Distribution of the Latent Roofs of the Covariance Matrix, Ann. Math. Statist 31, 151-158.

JAMEs, A. T. (1961). The Distribution of Nocentral Means with Known Covariance, Ann. Math. Statist. 32, 874-882.

JAMEs, A. T' (1961b). Zonal Polynomials of the Real Positive Definite Symmetric Matrices, Ann. Math. 74, 456-469.

JAMES, A. T. (1964). Distribution of Matrix Variates and Latent Roots Derived from Normal Samples, Ann. Math. Statist, 35, 475-501.

JAMES, A. T. (1968). Calculation of Zonal Polynomial Coefficients by the use of the Laplace-Beltrami Operator, Ann. Math. Statist. 39, 1711-1718.

Krishalal, P. R. (1960). Analysis of Variance, Handbook of Statistics, Vol. 1, P. R. Krishnaiah ed., North-Holland, Amsterdam.

Kshirsncinr, A. M. (1972). Multivariable Analysis. Dekker. New York.

LAWl.:Y. D. N. (1959). Test of Significance in Canonical Anal'sis, Biometrika 46, $59-66$.

MUtRhlind, R. J. (1982). Asperts of Multivariate Statistical Theory. John Wiley and Sons, Inc. New York.

PILLAI, K. C. S. (1956). On the Distribution of the Largest or the Smallest Root of a Matrix in Multivariate Analysis. Biometrika 43, $122-127$.

Pll.l.Al, K. C. S. (1967). On the Distribution of the Largest Root of a Matrix in Multivariate Analysis, Ann. Math. Statist. 38, 616-617.

Suglyama, T. (1967). Distribution of the Largest Latent Rood and the Smallest Laten Root of the Generalized B. Siatistic and F. Statistic in Multivariate. Analysis, Ann. Math. Statist. 38, 1152-1159.

Universidad Nacional de Tucumán

Facultad de Ciencias İ̃conómicas

lnstiuto de Investigaciones lstadisticas (INIE)

Casilla de Correo 209

4000 T'ueumár

Argentina 